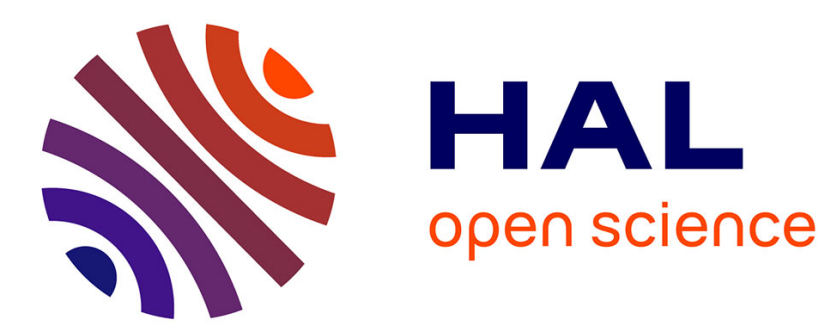

\title{
RADIS: A nonequilibrium line-by-line radiative code for CO2 and HITRAN-like database species
}

\author{
Erwan Pannier, Christophe Laux
}

\section{To cite this version:}

Erwan Pannier, Christophe Laux. RADIS: A nonequilibrium line-by-line radiative code for CO2 and HITRAN-like database species. Journal of Quantitative Spectroscopy and Radiative Transfer, 2019, 222-223, pp.12 - 25. 10.1016/j.jqsrt.2018.09.027 . hal-01904972

\section{HAL Id: hal-01904972 \\ https://hal.science/hal-01904972}

Submitted on 7 Feb 2020

HAL is a multi-disciplinary open access archive for the deposit and dissemination of scientific research documents, whether they are published or not. The documents may come from teaching and research institutions in France or abroad, or from public or private research centers.
L'archive ouverte pluridisciplinaire $\mathbf{H A L}$, est destinée au dépôt et à la diffusion de documents scientifiques de niveau recherche, publiés ou non, émanant des établissements d'enseignement et de recherche français ou étrangers, des laboratoires publics ou privés. 


\title{
RADIS: A Nonequilibrium Line-by-Line Radiative Code for $\mathrm{CO}_{2}$ and HITRAN-like database species
}

\author{
Erwan Pannier ${ }^{\mathrm{a}}$, Christophe O Laux ${ }^{\mathrm{a}}$ \\ ${ }^{a}$ Laboratoire EM2C, CNRS UPR288, CentraleSupélec, Université Paris Saclay, 3 rue Joliot Curie, 91190 \\ Gif sur Yvette
}

\begin{abstract}
An open-source nonequilibrium emission and absorption infrared line-by-line code is presented. RADIS is built around the HITRAN, HITEMP and CDSD databases for molecules in their electronic ground state. Energy levels are read from tabulated databases or calculated from Dunham expansions. Boltzmann, Treanor, and arbitrary vibrational distributions can be generated. In its present version, nonequilibrium spectra can be computed for $\mathrm{CO}_{2}$ and $\mathrm{CO}$, and equilibrium spectra can be computed for all species of the HITRAN database. New species can be added without modification to the core code. Several optimization strategies are described that reduce computation times of high-temperature, high-resolution spectra with millions of lines down to less than a minute. Additionally, RADIS includes a line survey tool, a built-in look-up database to reduce convergence times when fitting experimental spectra, and a multi-slab module to simulate line-of-sight experiments. Validation cases against existing spectral codes and experimental results from various plasma sources are presented. Finally, RADIS is applied to improve the modeling of the radiation from a plasma torch and a nonequilibrium expansion-tube experiment. RADIS is distributed under LGPLv3 license [1].
\end{abstract}

Keywords: Line-by-line code, nonequilibrium, optical emission spectroscopy, absorption spectroscopy

\section{Nomenclature}

$\mathrm{A}=$ Einstein emission coefficient, $\mathrm{s}^{-1}$

$\mathrm{j}_{\lambda}=$ spontaneous spectral emission density at wavelength $\lambda, \mathrm{W} / \mathrm{cm}^{3} / \mathrm{sr} / \mathrm{cm}^{-1}$

$\mathrm{E}=$ total rovibrational energy, $\mathrm{cm}^{-1}$

$\mathrm{E}_{\mathrm{rot}}=$ rotational energy, $\mathrm{cm}^{-1}$

$\mathrm{E}_{\mathrm{vib}}=$ vibrational energy, $\mathrm{cm}^{-1}$

Preprint submitted to Elsevier

October 11, 2018 
$\epsilon_{u l}=$ integral emission coefficient of transition $u \rightarrow l, \mathrm{~W} / \mathrm{cm}^{3} / \mathrm{sr}$

$\mathrm{g}_{\mathrm{vib}}=$ degeneracy of vibrational level

$\mathrm{g}_{\mathrm{i}}=$ state-independent degeneracy of a rotational level (function of the isotope)

$\mathrm{g}_{\mathrm{s}}=$ state-dependent degeneracy of a rotational level (function of the nuclear spin and the $\mathrm{J}$ parity)

$\mathrm{g}_{\mathrm{J}}=2 \mathrm{~J}+1$ degeneracy of rotational level $\mathrm{J}$ (associated to the $\mathrm{m}_{\mathrm{J}}$ number of the Zeeman states)

$\mathrm{h}=$ Planck's constant, $6.626 \times 10^{-34} \mathrm{~J} . \mathrm{s}$

$\mathrm{I}_{\lambda}=$ spectral radiance, $\mathrm{W} / \mathrm{cm}^{2} / \mathrm{sr} / \mathrm{cm}^{-1}$

$\mathrm{j}_{\lambda}=$ spectral emission density, $\mathrm{W} / \mathrm{cm}^{3} / \mathrm{sr} / \mathrm{nm}$

$\mathrm{J}=$ rotational quantum number

$\mathrm{k}_{\mathrm{b}}=$ Boltzmann constant, $1.381 \times 10^{-23} \mathrm{~J} / \mathrm{K}$

$\mathrm{k}_{\lambda}=$ spectral absorption coefficient, $\mathrm{cm}^{-1}$

$\mathrm{L}=$ path length of a homogeneous slab, $\mathrm{cm}$

$\lambda=$ wavelength, $\mathrm{nm}$

$\mathrm{n}=$ number density, $\mathrm{cm}^{-3}$

$\mathrm{n}_{\mathrm{u}}, \mathrm{n}_{\mathrm{l}}=$ number densities of the upper and lower states of a transition, $\mathrm{cm}^{-3}$

$\nu=$ wavenumber, $\mathrm{cm}^{-1}$

$\mathrm{p}=$ polyad number for polyatomic molecules (e.g. $2 \mathrm{v}_{1}+\mathrm{v}_{2}+3 \mathrm{v}_{3}$ for $\mathrm{CO}_{2}$ in this work)

$\Phi_{u l}=$ line lineshape for transition $u \rightarrow l$

$\mathrm{Q}_{\text {rovib }}=$ rovibrational partition function

$\mathrm{S}_{u l}=$ absorption linestrength for transition $l \rightarrow u, \mathrm{~cm} /$ molecule

$\mathrm{T}=$ translational temperature, $\mathrm{K}$

$\mathrm{T}_{\mathrm{vib}}, \mathrm{T}_{\text {rot }}=$ vibrational and rotational temperatures, $\mathrm{K}$

$\tau_{\lambda}=$ spectral transmittance

$\mathrm{v}_{\mathrm{i}}=$ vibrational level of vibrational mode $i$ in polyatomic molecules

$\mathrm{x}=$ mole fraction.

Gas compositions are expressed in mole fractions unless stated otherwise 


\section{Introduction}

This article introduces RADIS, a line-by-line (LBL) radiative solver to simulate the midIR emission spectra of $\mathrm{CO}_{2}$ and $\mathrm{CO}$ under nonequilibrium conditions, and the equilibrium spectra of any other species for which a HITRAN-type database is available. The RADIS solver can model a variety of vibrational population distributions, such as one and multitemperature Boltzmann distributions, Treanor distributions, or any arbitrary distribution. The code can also perform line-of-sight simulations of the spectra emitted by media with spatial gradients of species densities and temperatures. For these simulations, the radiative transfer equation is solved along multiple slabs, defined as homogeneous segments of gas. Several other radiation codes exist and are reviewed below. As seen from this review, few of them are capable of predicting spectra with a variety of nonequilibrium distributions and with inhomogeneous line-of-sight conditions.

Specair $[2,3]$ is widely used to predict the emission and absorption spectra of many atomic and diatomic species with $\mathrm{N}, \mathrm{O}, \mathrm{C}$, and $\mathrm{H}$ elements but its current version (3.0) does not include triatomic species such as $\mathrm{CO}_{2}$. It is a useful tool to analyze experimental spectra, with nonequilibrium distributions and line-of-sight distributions (although the commercial version 3.0 is limited to one slab). Specair can also convolve theoretical spectra with the instrumental broadening function, which is critical for fitting experimental spectra. In this work, Specair was used to validate the calculation of nonequilibrium partition functions as detailed in section 5, and to verify the proper implementation of instrumental broadening in RADIS.

SpectraPlot.com [4] is a web-based tool that uses the NIST ASD and HITRAN databases, with a handy line survey feature. It can model $\mathrm{CO}_{2}$ spectra, but the current version does not calculate line-of-sight spectra, nor arbitrary nonequilibrium spectra. HAPI, the HITRAN Python interface [5], is also limited to equilibrium spectra. MassiveOES [6] is an open source software that features arbitrary vibrational distributions, but does not include $\mathrm{CO}_{2}$ yet.

Klarenaar et al. [7] developed a nonequilibrium $\mathrm{CO}_{2}$ code with Boltzmann rotational distributions $\left(\mathrm{T}_{\text {rot }}\right)$ and mode-specific Treanor vibrational distributions $\left(\mathrm{T}_{\mathrm{vib} 1,2,3}\right)$. They used line positions and line intensities from the HITEMP-2010 database, and calculated populations with rovibrational energies based on two-term Dunham expansions for vibration $\left(\omega_{\mathrm{e}}, \omega_{\mathrm{e}} \mathrm{x}_{\mathrm{e}}\right)$ and three-term expansions for rotation $(\mathrm{B}, \mathrm{D}, \mathrm{H})$, without vibration-rotation coupling terms. However, the code is currently limited to absorption. A comparison between this code and RADIS is used in the validation procedure of section 5 .

NEQAIR [8], the NASA LBL code, computes nonequilibrium mid-IR $\mathrm{CO}_{2}$ radiation $[9,10]$ on the basis of the CDSD-4000 database for line positions, intensities, and energy levels [11]. The CDSD-4000 database is fitted on experimental data and includes rotationalvibrational coupling, Fermi and Coriolis interactions, and l-doubling of rovibrational energy 
levels [12]. The CDSD-4000 database is valid for temperatures of up to $5000 \mathrm{~K}$ and thus includes 626 million lines. In NEQAIR, the CDSD-4000 database is reduced by merging weak lines in a tabulated pseudo-continuum, which allows drastically reduced computation times while preserving the accuracy at high temperature. However, to our knowledge, NEQAIR uses Boltzmann distributions for the rotational and vibrational distributions, and does not include Treanor distributions. HARA [13], the other NASA LBL code, also includes mid-IR $\mathrm{CO}_{2}$ radiation [14] based on the CDSD-4000 database.

Rivière and Soufiani [15] used $\mathrm{CO}_{2}$ LBL code based on the CDSD-4000 database to generate statistical narrow-band and correlated-k models for $\mathrm{CO}_{2}$ in the infrared. JAXA included $\mathrm{CO}_{2}$ modeling in the SPRADIAN radiation code. So far only equilibrium results [16] have been published although work is underway to simulate nonequilibrium spectra [17]. The PASTIS [18] radiation code, developed to study the emission of a nonequilibrium $\mathrm{CO}_{2}$ plasma, is limited to the $\mathrm{CO}_{2}$ dissociation products and does not include $\mathrm{CO}_{2}$ itself. PARADE is a nonequilibrium LBL code that was extended to include $\mathrm{CO}_{2}$ [19]. SPARTAN [20] is another LBL code that features $\mathrm{CO}_{2}$. It was used to study $\mathrm{CO}_{2}-\mathrm{N}_{2}$ plasmas [21] under equilibrium, but it also includes a reduced dataset based on CDSD that can be used to simulate the IR emission of nonequilibrium $\mathrm{CO}_{2}$.

Finally, many nonequilibrium, state-specific $\mathrm{LBL} \mathrm{CO}_{2}$ radiation codes can be found in the atmospheric sciences community. For instance, groups working on the simulation of Mars atmosphere radiation (where $\mathrm{CO}_{2}$ is excited by the solar flux, as measured by space experiments such as MarsExpress), developed nonequilibrium LBL codes for $\mathrm{CO}_{2}[22,23]$. These codes usually have fully coupled collisional-radiative (CR) models, i.e. the vibrational populations are calculated by coupling radiation and collisional processes. In our case studies, excited state populations remain low, the plasma discharges are mostly optically thin, and populations distributions are governed by collisional processes only, especially at atmospheric pressure. Hence, the effect of radiation on the vibrational populations can be neglected, and such fully coupled CR codes are not needed.

This paper presents the LBL code RADIS and several validation case studies. RADIS was initially developed to study the vibrational excitation of carbon dioxide by nanosecond pulsed discharges. Phase-locked, time-resolved spectra were measured in the $4.2-5 \mu \mathrm{m}$ spectral range. These results will be presented in a future work [24], however most of the examples of section 3 and the validation cases of section 5 correspond to this spectral range. In the next section, we present the physical models used in RADIS, in particular regarding the energy levels used for each molecule. Section 3 describes the various strategies implemented to improve computational performance. In Section 4, the multi-slab solver is presented. In Section 5, we validate the code against calculations from HAPI and Specair, and experimental data from a $\mathrm{CO}_{2}$ laser, a Microwave plasma torch and a shock-tube experiment. RADIS is finally applied to improve the spectral modeling of an equilibrium RF torch [25]. 


\section{Spectral code}

RADIS was written as a general purpose radiative solver for rovibrational emission and absorption transitions. It allows for arbitrary rovibrational distributions of $\mathrm{CO}_{2}$ and $\mathrm{CO}$, and features a multi-slab solver. RADIS can be easily extended to other species thanks to its modular architecture. Several tools were also developed to fit experimental spectra, in particular a line survey tool and a built-in interface to a look-up database of pre-calculated spectra to increase convergence speed in spectral fits. These tools are described in the User Documentation [1]. The code is distributed under a GNU Lesser General Public License (LGPLv3), i.e. it is available for use and modifications [1].

Line positions are taken from several line databases such as HITRAN, HITEMP [26], and CDSD-4000, which can be swapped for comparison purposes. As an example, the line position databases of HITEMP-2010 and CDSD-4000 are compared in the high temperature validation case of section 5. Line intensities are determined from Einstein A coefficients tabulated in the databases, and calculated rovibrational populations. Absorption linestrengths are scaled from reference linestrengths as detailed in the Appendix of Rothman et al. [27]. Rovibrational populations can follow one-temperature (equilibrium), two-temperature $\left(\mathrm{T}_{\mathrm{vib}}\right.$, $\left.\mathrm{T}_{\text {rot }}\right)$ or multiple-temperature $\left(\mathrm{T}_{\mathrm{vib1}, 2,3}, \mathrm{~T}_{\text {rot }}\right.$ for $\left.\mathrm{CO}_{2}\right)$ distributions. Vibrational populations can follow either Boltzmann or Treanor distributions, or arbitrary distributions by using vibrational specific nonequilibrium factors. Rotational populations, however, are always assumed to follow a Boltzmann distribution $\left(\mathrm{T}_{\mathrm{rot}}\right)$. The energies of the emitting or absorbing rovibrational levels are taken from the aforementioned line databases. Partition functions are either interpolated from tabulated databases (TIPS Fischer et al. [28], CDSD Tashkun and Perevalov [11]), or calculated directly by summing over all levels. In the latter case, the rovibrational level energies are either taken from energy databases (CDSD-4000 [29]), or calculated from Dunham expansions. More details regarding the energy levels used for each molecule can be found in section 2.5.

Figure 1 shows a schematic of the structure of RADIS. The following sections present the calculation of line positions, line intensities, broadening widths, energies, and populations.

\subsection{Line positions}

Line positions are calculated from tabulated data, with a correction for pressure shift using the coefficients given in the line databases. For measurements in air, the wavelength is corrected with the refractive index of air from Ciddor [30]. 


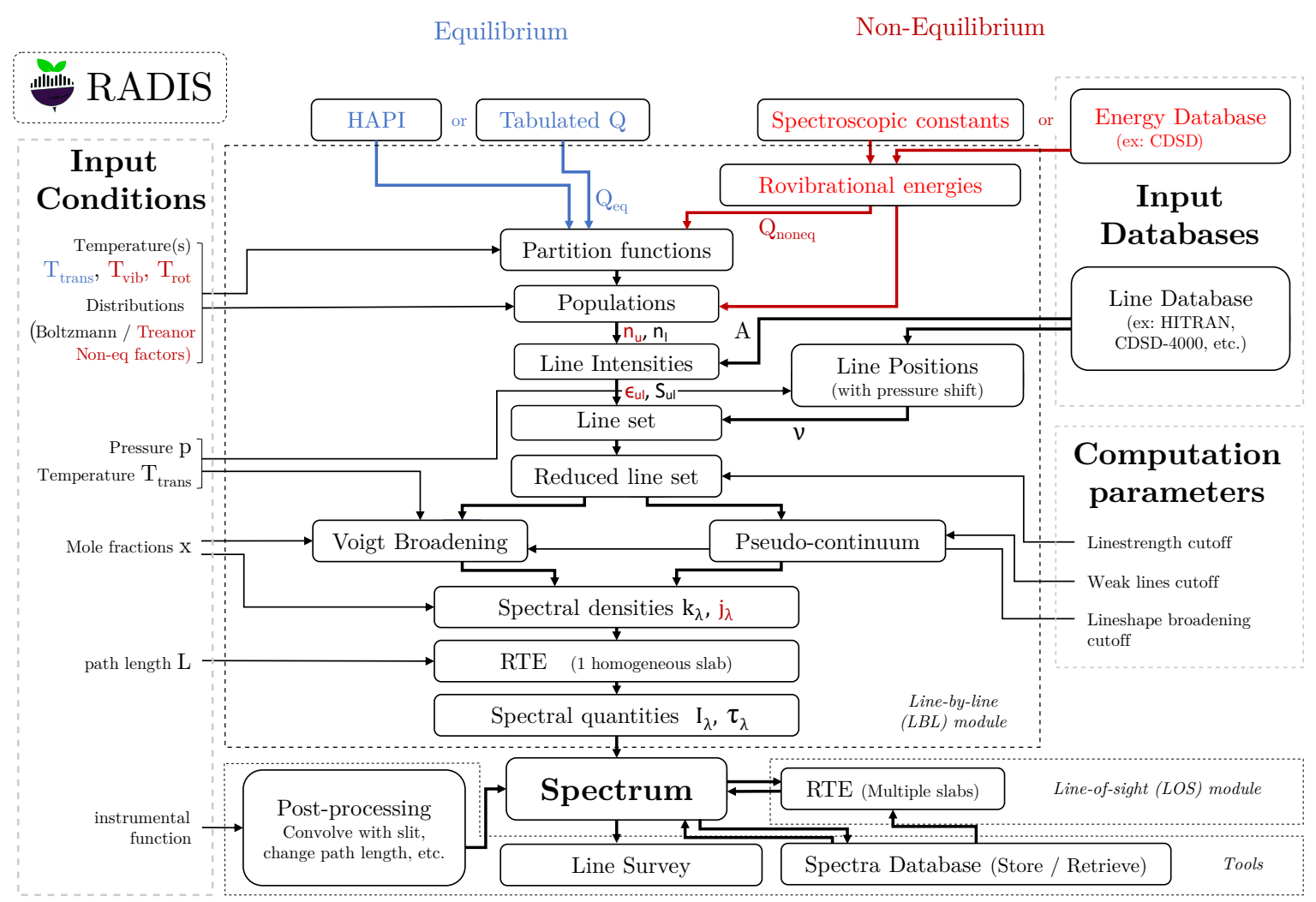

Figure 1: Flow chart of the RADIS spectral code

\subsection{Line intensities}

Under equilibrium conditions, the linestrengths at temperature $\mathrm{T}$ are calculated from the tabulated linestrengths at temperature $\mathrm{T}_{\text {ref }}$ of the line database by correcting for the new population distribution (Boltzmann distribution at $\mathrm{T}$ ), partition functions (tabulated), and stimulated emission, as explained in the Appendix of [27]. The linestrengths are multiplied by the lineshape function (accounting for Doppler and collisional broadening), and a summation is performed over all lines to get the spectral absorption coefficient $k_{\lambda}$. The spectral absorption coefficient is then multiplied by the path length to get the spectral absorbance, or optical density, from which the spectral transmittance $\tau_{\lambda}$ is calculated. The spectral emissivity is calculated from the spectral transmittance using Kirchhoff's law, and multiplying by the Planck function yields the spectral radiance $I_{\lambda}$.

Under nonequilibrium conditions, the absorption coefficient is still calculated from tabulated linestrengths, but nonequilibrium populations and partition functions are calculated from the rovibrational energy of each level. The radiance is obtained from the Einstein emission 
coefficient A, by first computing the integral emission coefficient (typically in $\mathrm{W} / \mathrm{cm}^{3} / \mathrm{sr}$ ):

$$
\epsilon_{\mathrm{ul}}=n_{u} \frac{A_{\mathrm{ul}}}{4 \pi} \Delta \epsilon_{\mathrm{ul}}
$$

Einstein coefficients are usually given in the HITRAN-like databases, or can be recomputed from the weighted transition moment squared $\mathfrak{R}_{\mathrm{ul}}$ which can be inferred from the linestrength [27]. The integral emission coefficient is broadened with the lineshape $\Phi$ and summed over all lines to get the spectral emission density $\mathrm{j}_{\nu}$ :

$$
j_{\nu}=\sum_{\mathrm{ul}} j_{\mathrm{ul}}(\nu)=\sum_{\mathrm{ul}} \epsilon_{\mathrm{ul}} \Phi_{\mathrm{ul}}(\nu)
$$

In RADIS, the emission density is calculated in the wavenumber space $\left(\mathrm{j}_{\nu}\right.$ in $\left.\mathrm{W} / \mathrm{cm}^{3} / \mathrm{sr} / \mathrm{cm}^{-1}\right)$, and is then converted to the wavelength space $\left(\mathrm{j}_{\lambda}\right.$ in $\left.\mathrm{W} / \mathrm{cm}^{3} / \mathrm{sr} / \mathrm{nm}\right)$. The spectral radiance corrected for self-absorption, $I_{\lambda}\left(\mathrm{W} / \mathrm{cm}^{2} / \mathrm{sr} / \mathrm{nm}\right)$, is obtained by solving analytically the 1-D Radiative Transfer Equation (RTE) for a homogeneous column of gas of length L. When scattering is neglected, we obtain:

$$
I_{\lambda}=\int_{L} j_{\lambda} \exp \left(-k_{\lambda}(L-x)\right) \mathrm{dx}
$$

which finally yields the spectral radiance of the slab:

$$
I_{\lambda}=j_{\lambda} \frac{1-\exp \left(-k_{\lambda} L\right)}{k_{\lambda}}
$$

\subsection{Line broadening}

The lineshape $\Phi(\nu)$ of a transition is determined by considering Doppler, resonant, and van der Waals broadenings. Other broadening mechanisms such as Stark broadening are not yet implemented. Resonant and van der Waals broadenings in RADIS are calculated from tabulated collisional broadening half-widths at half-maximum (HWHM) and correction coefficients for temperature, as detailed in [27], Appendix. These coefficients are available in the various HITRAN-like line databases used in this work. By default, the van der Waals broadening HWHM is that in air. When the gas composition differs from air (e.g. for CO lines in a gas composed mostly of $\mathrm{CO}_{2}$, as in our case study), the air broadening coefficients (and associated correction coefficients) should be replaced by the broadening coefficients of the main gas component (e.g. $\mathrm{CO}_{2}$ ), which are usually available from the HITRAN database.

The broadened lineshape is implemented using the empirical approximation of the Voigt profile of Whiting [31], and the equivalent Voigt width of Olivero and Longbothum [32]. Whiting uses an empirical polynomial fit of the Voigt area to normalize the lineshape. In RADIS, the line shape is normalized numerically in all cases to ensure energy conservation (even if the spectral grid is too sparse to capture the line shape correctly). 


\subsection{Distributions}

In RADIS, all rotational levels follow a Boltzmann distribution at temperature $\mathrm{T}_{\text {rot }}$ with degeneracy $g_{\text {rot }}=g_{s} g_{i} g_{J}$ where $g_{s}, g_{i}, g_{J}$ are the state-dependent, state-independent, and $(2 J+1)$ degeneracy of the rotational levels. The degeneracies $g_{s}$ and $g_{i}$ depend on the quantum rules that characterize a given isotope, and can be found for HITRAN molecules and isotopes in Ref. [33].

Vibrational levels can follow a Boltzmann distribution at temperature $\mathrm{T}_{\mathrm{vib}}$. The vibrational Boltzmann distribution is expressed with the vibrational degeneracy $g_{\text {vib }}$ ( 1 for vibrational levels of $\Sigma$ symmetry) and the vibrational energy $\mathrm{E}_{\mathrm{vib}}$ :

$$
\frac{n_{\mathrm{vib}}}{n_{\mathrm{tot}}}=\frac{g_{\mathrm{vib}}}{Q_{\mathrm{vib}}\left(T_{\mathrm{vib}}\right)} \exp \left(-\frac{E_{\mathrm{vib}}}{k T_{\mathrm{vib}}}\right)
$$

Vibrational levels can also follow a Treanor distribution, which better accounts for rotational - vibrational coupling in high vibrational levels, and is valid when VV relaxation processes are much faster than VT relaxation processes. The Treanor distribution is expressed with the harmonic $\mathrm{E}_{\mathrm{vib}}^{\mathrm{h}}$ and anharmonic $\mathrm{E}_{\mathrm{vib}}^{\mathrm{a}}$ components of the vibrational energy:

$$
\frac{n_{\mathrm{vib}}}{n_{\mathrm{tot}}}=\frac{g_{\mathrm{vib}}}{Q_{\mathrm{vib}}\left(T_{\mathrm{vib}}, T_{\mathrm{rot}}\right)} \exp \left(-\left(\frac{E_{\mathrm{vib}}^{h}}{k T_{\mathrm{vib}}}+\frac{E_{\mathrm{vib}}^{a}}{k T_{\mathrm{rot}}}\right)\right)
$$

In RADIS, Treanor distributions can be calculated if the vibrational energy can be separated into harmonic and anharmonic components. For instance, for a diatomic molecule, if energies are fitted with a third-order Dunham expansion, the harmonic and anharmonic energies are $\mathrm{E}_{\mathrm{vib}}^{\mathrm{h}}=\omega_{\mathrm{e}}(\mathrm{v}+1 / 2)$ and $\mathrm{E}_{\mathrm{vib}}^{\mathrm{a}}=-\omega_{\mathrm{e}} \mathrm{x}_{\mathrm{e}}(\mathrm{v}+1 / 2)^{2}+\omega_{\mathrm{e}} \mathrm{y}_{\mathrm{e}}(\mathrm{v}+1 / 2)^{3}$, respectively.

For polyatomic molecules, different vibrational temperatures $\mathrm{T}_{\mathrm{vib}, \mathrm{i}}$ can be assumed for all of the vibration modes. For instance, $\mathrm{CO}_{2}$ can be described with up to three vibrational temperatures, corresponding to the symmetric $\omega_{1}$, bending $\omega_{2}$ and asymmetric $\omega_{3}$ vibration modes, even if is often assumed that $\mathrm{T}_{\mathrm{vib}, 1}=\mathrm{T}_{\mathrm{vib}, 2}$ owing to strong coupling between the symmetric and bending modes [34, 35, 36]. In RADIS, the population distributions are then calculated for each vibrational mode using the mode energy $E_{v i b, i}$ (Boltzmann) or $E_{v i b, i}^{\mathrm{h}}$, $\mathrm{E}_{\mathrm{vib}, \mathrm{i}}^{\mathrm{a}}$ (Treanor).

Certain specific cases require non-Boltzmann or non-Treanor vibrational state distributions. In RADIS, this is implemented by applying a state-specific nonequilibrium factor $\alpha_{\mathrm{v}}$ to a Boltzmann or Treanor distribution. For instance, the nonequilibrium vibrational population of level v may be defined relative to a one-temperature Boltzmann distribution as follows:

$$
\frac{n_{v}(v)}{n_{\text {tot }}}=\alpha_{v} \frac{g_{v}}{Q_{\text {vib }}\left(T_{\text {vib }}\right)} \exp \left(-\frac{E_{v}}{8}\right)
$$


Note that if such a vibrational population distribution is used, the partition function is modified to ensure conservation of the total molecular number density. For example, in the case of the distribution defined by Eqn. 2.7, the partition function is given by:

$$
Q_{\mathrm{vib}}=\sum_{v} \alpha_{v} g_{v} \exp \left(-\frac{E_{v}}{\mathrm{kT}_{\mathrm{vib}}}\right)
$$

\subsection{Energies}

To calculate nonequilibrium population distributions, the energies of all rovibrational levels are needed. Energy levels can either be provided as inputs to RADIS, or calculated from built-in spectroscopic constants. Energies are then calculated from Dunham expansions of a rotating vibrator model, that can be either coupled if rotational-vibrational interaction terms are taken into account (for instance, the usual $\beta_{\mathrm{e}}$ coefficient corrects the centrifugal term $\mathrm{D}_{\mathrm{e}}$ for the effect of vibration), or uncoupled if rotation and vibration are considered independent. Currently, RADIS incorporates built-in spectroscopic constants for $\mathrm{CO}$ and $\mathrm{CO}_{2}$, which are described below. The vibration-rotational interaction terms of Dunham expansions appear as diagonal terms in the rovibrational Hamiltonian. To include nondiagonal interactions terms, such as Fermi coupling, Coriolis coupling and l-doubling, a database of energy levels precomputed with these interaction must be supplied to RADIS as an Energy Database (see Figure 1).

Carbon monoxide. For vibrational numbers $\mathrm{v} \leq 17$, the rovibrational energy levels of $\mathrm{CO}$ are calculated with a coupled rotating vibrator model using Dunham expansions:

$$
E(v, J)=\sum_{i, j} Y_{i, j}\left(v+\frac{1}{2}\right)^{i}(J(J+1))^{j}
$$

The $\mathrm{Y}_{\mathrm{ij}}$ spectroscopic constants are taken from Ref. [37], which includes interaction terms for seven isotopes of $\mathrm{CO}$. The energies calculated with these constants are verified to match these of Ref. [38, 39] within $0.1 \%$ for the main isotope ${ }^{12} \mathrm{C}^{16} \mathrm{O}$. For higher vibrational numbers, a Morse potential is used up to level $\mathrm{v}=48$. Rotational levels are calculated up to the dissociation limit $(11.16 \mathrm{eV})$.

Carbon dioxide. The built-in spectroscopic constants reviewed by Klarenaar et al. [7] were implemented for the three main isotopes of $\mathrm{CO}_{2}$, and were used to reproduce the validation case of Figure 13 in section 5. The rovibrational energies are then calculated with an uncoupled vibrating rotor model, using a first order anharmonicity correction for each of the three vibration modes considered $\left(\omega_{\mathrm{e}} \mathrm{x}_{\mathrm{e} 1}, \omega_{\mathrm{e}} \mathrm{x}_{\mathrm{e} 2}, \omega_{\mathrm{e}} \mathrm{x}_{\mathrm{e} 3}\right)$, and a second order correction for the centrifugal force $(\mathrm{D}, \mathrm{H})$. For consistency with the calculation of rovibrational energies 
of diatomic molecules, the Dunham formalism of Eqn. 2.9 is adapted to $\mathrm{CO}_{2}$ with $\mathrm{Y}_{\mathrm{i}_{1} \mathrm{i}_{2} \mathrm{i}_{3} \mathrm{j}}$ coefficients in Eqn. 2.10:

$$
\left(v_{1}, v_{2}, v_{3}, J\right)=\sum_{i_{1} i_{2} i_{3}, j} Y_{i_{1} i_{2} i_{3} j}\left(v_{1}+\frac{1}{2}\right)^{i_{1}}\left(v_{2}+1\right)^{i_{2}}\left(v_{3}+\frac{1}{2}\right)^{i_{3}}(J(J+1))^{j}
$$

The uncoupled vibrating rotor model mentioned above corresponds to $\mathrm{Y}_{0001}=\mathrm{B}, \mathrm{Y}_{0002}=\mathrm{D}$, $\mathrm{Y}_{0003}=\mathrm{H}, \mathrm{Y}_{1000}=\omega_{1}, \mathrm{Y}_{0100}=\omega_{2}, \mathrm{Y}_{0010}=\omega_{3}, \mathrm{Y}_{2000}=-\omega_{\mathrm{e}} \mathrm{x}_{\mathrm{e} 1}, \mathrm{Y}_{0200}=-\omega_{\mathrm{e}} \mathrm{x}_{\mathrm{e} 2}, \mathrm{Y}_{0020}=-\omega_{\mathrm{e}} \mathrm{x}_{\mathrm{e} 3}$, and all other $\mathrm{Y}_{\mathrm{i}_{1} \mathrm{i}_{2} \mathrm{i}_{3} \mathrm{j}}=0$. This model includes neither vibrational-rotational (v-J) coupling, Fermi coupling, Coriolis coupling, nor l-doubling perturbations. Furthermore, the low-order polynomial fit can fail to reproduce the energies of higher lying levels that become populated at high temperatures.

To assess the effect of interaction terms on the calculated spectra, emission spectra and partition functions of the first isotope ${ }^{12} \mathrm{C}^{16} \mathrm{O}_{2}$ were compared to emission spectra and partition function calculated with the rovibrational energies used in CDSD-4000 [29]. These energies were calculated by S.A.A Tashkun from the effective Hamiltonian of Tashkun et al. [12], which uses high order polynomial expansions, and includes v-J coupling, Fermi coupling, Coriolis coupling, and l-doubling. At equilibrium, the uncoupled vibrating rotor of Eqn. 2.10 was found to yield the same partition functions as the one of the CDSD-4000 database at up to $6000 \mathrm{~K}$, which suggest that rovibrational energies are very similar in both cases. Nonequilibrium calculations, however, present other issues which are addressed in the next section.

\subsection{Coupling terms under nonequilibrium}

In a nonequilibrium model where $\mathrm{T}_{\mathrm{vib}} \neq \mathrm{T}_{\text {rot }}$ the inclusion of coupling terms raises another question: shall they be assigned to the vibrational or to the rotational energy? For diatomic molecules, this question has been addressed by Babou et al. [40], who wrote the total rovibrational energy as the sum of the uncoupled energies for each energy mode $\bar{E}_{\mathrm{vib}}, \bar{E}_{\mathrm{rot}}$, and an interaction terms $\mathrm{E}_{\text {coupling: }}$ :

$$
E(v, J)=\bar{E}_{\mathrm{vib}}(v, J)+\bar{E}_{\mathrm{rot}}(v, J)+E_{\text {coupling }}(v, J)
$$

Babou et al. [40] quantified the effect of coupling terms on nonequilibrium partition function in two opposite scenarios, where the coupling energy was assigned to either the vibrational or the rotational terms. They observed no significant difference for diatomic molecules at temperatures of up to $10,000 \mathrm{~K}$.

For $\mathrm{CO}_{2}$, coupling terms can be more important than for diatomic molecules. This effect is studied separately in future work [41], where we investigate different nonequilibrium models 
for the assignation of vibrational and rotational energies in $\mathrm{CO}_{2}$ : the uncoupled vibrating rotor model of Eqn. 2.10 is compared with the NEQAIR nonequilibrium $\mathrm{CO}_{2}$ model [10], based on a parametrization of the CDSD-4000 energies. As a reference, the effective Hamiltonian of [12] is implemented and diagonalized to extract rotational, vibrational and coupling components. Different energy databases are then compared with coupling terms assigned either to the vibrational or to the rotational energy. The preliminary results show that the impact of coupling terms for $\mathrm{CO}_{2}$ is larger than for diatomic molecules, but that for conditions with $T_{\text {rot }}{ }^{0.9}<T_{\text {vib }}<T_{\text {rot }}{ }^{1.1}$, and $T_{\text {vib }}<10,000 \mathrm{~K}$, the uncoupled model of Eqn. 2.10 may be used safely [41].

\subsection{Partition functions}

The line databases considered in the present work are all limited to the ground electronic state of molecules. Therefore, the partition functions are approximated to the rovibrational partition functions of ground state molecules. This is valid for $\mathrm{k}_{\mathrm{b}} \mathrm{T} \ll \mathrm{E}_{\text {elec }}$, where $\mathrm{E}_{\text {elec }}$ is the energy of the first excited electronic state. For $\mathrm{CO}_{2}$ and diatomic molecules, the energy of the first excited state is typically $\mathrm{E}_{\text {elec }} \sim 3-5 \mathrm{eV}$ [42]. Hence, this approximation is valid for the validations cases presented here $(\mathrm{T} \leq 3500 \mathrm{~K})$.

In RADIS, at equilibrium, partition functions are interpolated from tabulated databases, for instance using HITRAN through the HAPI interface [5]. Under nonequilibrium conditions, partition functions are calculated by summing over all levels. In a two-temperature model $\left(\mathrm{T}_{\mathrm{vib}}, \mathrm{T}_{\text {rot }}\right)$, if coupling is neglected, then the nonequilibrium partition function can be written as the product of the vibrational and rotational partition functions:

$$
Q_{\text {rovib }}\left(T_{\mathrm{vib}}, T_{\mathrm{rot}}\right)=Q_{\mathrm{vib}} \cdot Q_{\mathrm{rot}}=\left(\sum_{v} g_{v} e^{-\frac{E_{\mathrm{vib}}(v)}{\mathrm{kT}}}\right) \cdot\left(\sum_{J} g_{s} g_{i} g_{J} e^{-\frac{E_{\mathrm{rib}}(J)}{k T_{\mathrm{rot}}}}\right)
$$

When coupling is considered, this approximation does not hold and only the total rovibrational partition function is defined:

$$
Q_{\text {rovib }}\left(T_{\text {vib }}, T_{\text {rot }}\right)=\sum_{v}\left[\mathrm{~g}_{\mathrm{v}} e^{-\frac{E_{\mathrm{vib}}(v, J)}{\mathrm{kT} \mathrm{T}_{\mathrm{vib}}}}\left(\sum_{J} g_{s} g_{i} g_{J}(v) e^{-\frac{E_{\mathrm{rot}}(v, J)}{k T_{\mathrm{rot}}}}\right)\right]
$$

For $\mathrm{CO}_{2}$, even if the energies of the uncoupled vibrating rotor model are used, Eqn. 2.12 cannot be used when the bending levels $\mathrm{v}_{2} \leq 1$ are significantly populated $\left(\mathrm{T}_{2} \geq 1000 \mathrm{~K}\right)$, because the rotational levels $\mathrm{J} \leq \mathrm{l}_{2}$ of a bent level $\mathrm{l}_{2}>0$ do not exist, i.e. the rotational degeneracy $\mathrm{g}_{\mathrm{J}}$ depends on the vibrational level $\mathrm{v}$ : $\mathrm{g}_{\mathrm{J}}=2 \mathrm{~J}+1$ if $\mathrm{J} \leq \mathrm{l}_{2}$ and $\mathrm{g}_{\mathrm{J}}=0$ if $\mathrm{J}<\mathrm{l}_{2}$. In that case, only $Q_{\text {rovib }}$ of Eqn. 2.13 is defined. 


\section{Computation parameters}

\subsection{Implementation}

RADIS is written in Python, making use of C-compiled libraries (NumPy, Numba) for computationally intensive steps, and data analysis libraries (Pandas) to handle lines databases efficiently. In a typical LBL code, the lineshape broadening step (section 2.3) is usually the most computationally expensive calculation step. To improve performance, RADIS features a built-in CPU parallelization of the lineshape broadening step. In the following, however, all numbers regarding computation performance are given for one CPU core. Database loading was also an important performance bottleneck as line databases can weigh dozens of gigabytes, and has been optimized with HDF5 support. The computation times in RADIS are comparable with those of state-of-the-art Fortran codes. However, when calculating spectra of polyatomic molecules at high temperature $(>2000 \mathrm{~K})$ and over wide spectral ranges, millions of lines are necessary and computation times increase drastically. Using the optimization strategies detailed in the next sections, the computation speed in RADIS can be further increased by orders of magnitude.

\subsection{Linestrength cutoff criteria}

To improve computational performance, the lines whose linestrength after temperature scaling is below a certain cutoff are discarded. Figure 2 shows the convergence of the cumulated linestrength for all lines of a spectrum as a function of the linestrength cutoff for the typical case of the $\mathrm{CO}_{2} 4.2 \mu \mathrm{m}$ bands between 2050 and $2400 \mathrm{~cm}^{-1}$. The number of lines above the cutoff limit, also shown in Fig. 2, is a good metric of the computation time. As a rule of thumb, computation requires about $100 \mathrm{~ns} /$ line/spectral point on the test configuration used (Section 3.6). Under equilibrium and optically thin conditions, the cumulated linestrength is a good metric of the accuracy of the emission spectrum calculated. When the spectrum becomes optically thicker, the relative importance of small lines increases and the linestrength cutoff should be reduced. In extreme nonequilibrium cases, a cutoff on the integral emission coefficient (Eqn. 2.1) should be used to calculate emission spectra. In the example of Fig. 2, linestrengths were computed at three temperatures (300, 1000 and $3500 \mathrm{~K}$ ) using the CDSD-4000 database. The cutoff necessary to obtain at least $99 \%$ of the total cumulated linestrength is shown (dashed lines). At 300, 1000 and $3500 \mathrm{~K}$, it is required to resolve 180, 13k and 2.6M lines, respectively. Computation times increase proportionally: with a spectral resolution of $0.01 \mathrm{~cm}^{-1}$ and a lineshape broadening of $10 \mathrm{~cm}^{-1}, 1000$ spectral points are required, which, as per our rule of thumb (100 ns/line/spectral point), yields respective computation times of $0.2 \mathrm{~s}, 13 \mathrm{~s}$, and $40 \mathrm{~min}$, if no additional optimization is performed.

Unless stated otherwise, the default linestrength cutoff in RADIS is $10^{-27} \mathrm{~cm} / \mathrm{molecule}$. Figure 2 shows that an increase of this cutoff can yield improvements of several orders of 


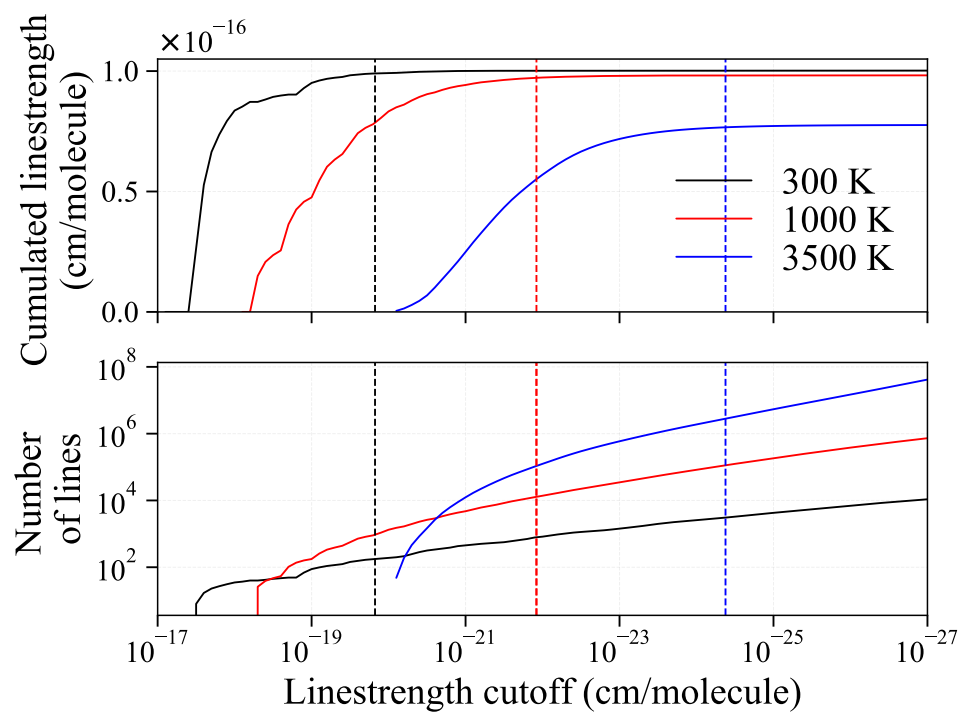

Figure 2: Cumulated linestrength (estimate of the spectrum accuracy) and number of lines (estimate of the computational time) as a function of the linestrength cutoff for three different temperatures (solid, black: $300 \mathrm{~K}$, red: $1000 \mathrm{~K}$, blue: $3500 \mathrm{~K})$, for $\mathrm{CO}_{2}$ in the spectral range $2050-2400 \mathrm{~cm}^{-1}(4.2 \mu \mathrm{m}$ band), and cutoff above $99 \%$ of the cumulated linestrength is calculated (dashed, black: $300 \mathrm{~K}$, red: $1000 \mathrm{~K}$, blue: $3500 \mathrm{~K}$ )

magnitude on performance with a minimal impact on the spectrum, until a critical value is reached, which increases with higher temperatures. Figures 3 and 4 corroborate this by showing the effect of increasing the linestrength cutoff on the $4.2 \mu \mathrm{m}$ emission band of $\mathrm{CO}_{2}$ for two temperatures, $2200 \mathrm{~K}$ and $3409 \mathrm{~K}$, as calculated with the HITEMP-2010 database. Temperatures and mole fractions correspond to the validation case of section 5.6. The difference between the two spectra is particularly visible in the band tails, where lines involve less populated, high energy levels. This difference is amplified at lower temperatures as fewer lines exceed the linestrength cutoff. The maximum relative difference between the two spectra reaches $10 \%$ below $2050 \mathrm{~cm}^{-1}$ at $3409 \mathrm{~K}$, and $10 \%$ below $2150 \mathrm{~cm}^{-1}$ at $2200 \mathrm{~K}$.

\subsection{Influence of the line database}

The effect of the linestrength cutoff criterion presented above changes with different line databases. Line databases such as HITRAN or CDSD-4000 are typically generated with a linestrength cutoff of $10^{-27} \mathrm{~cm} /$ molecule at a specified reference temperature (e.g. $4000 \mathrm{~K}$ for CDSD-4000). In Figure 5 and 6, spectra are calculated for temperatures of 2200 and $3409 \mathrm{~K}$ with CDSD-4000 [11] and HITEMP-2010 [26], whose reference temperature (1000 K) is lower than that of CDSD-4000.

At $2200 \mathrm{~K}$ both spectra agree. At $3409 \mathrm{~K}$, however, the HITEMP-2010 database differs significantly from the CDSD-4000 reference in the hot bands below $2200 \mathrm{~cm}^{-1}$. Thus, in section 5 , the validation cases at temperatures below $2200 \mathrm{~K}$ are calculated with HITEMP- 


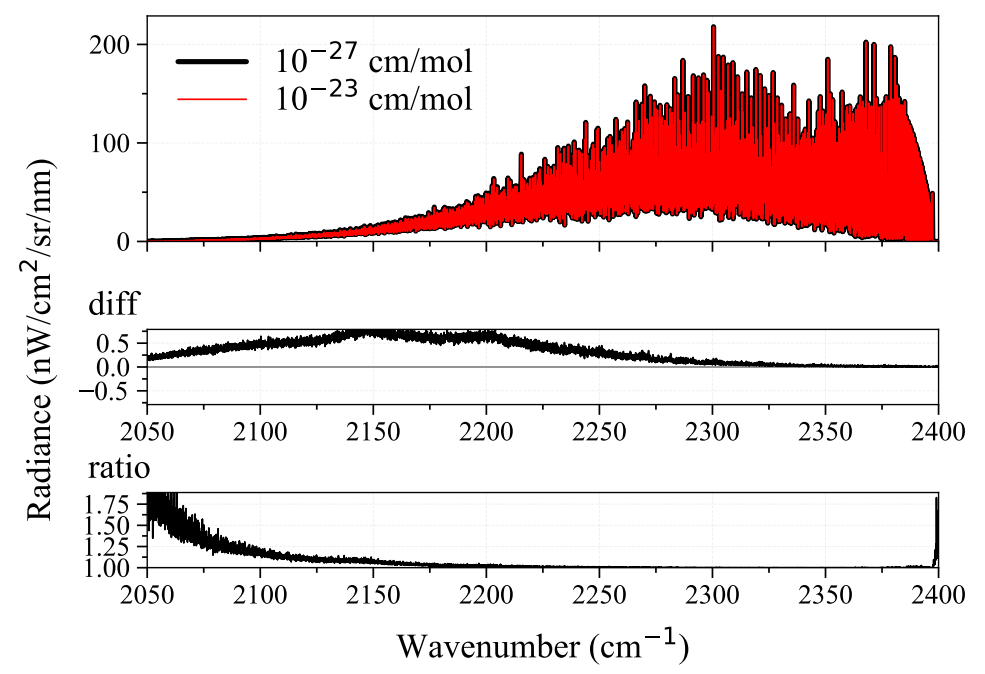

Figure 3: Effect of the linestrength cutoff on the emission spectrum of the $4.2 \mu \mathrm{m}$ band of $\mathrm{CO}_{2}$ under the following conditions: $\mathrm{T}=2200 \mathrm{~K}, \mathrm{p}=1 \mathrm{~atm}, \mathrm{x}_{\mathrm{CO}_{2}}=3.3 \times 10^{-4}, \mathrm{~L}=0.05 \mathrm{~cm}$, broadening cutoff: $50 \mathrm{~cm}^{-1}$, HITEMP-2010 database. Red line: $10^{-23} \mathrm{~cm} /$ molecule, black line: $10^{-27} \mathrm{~cm} /$ molecule

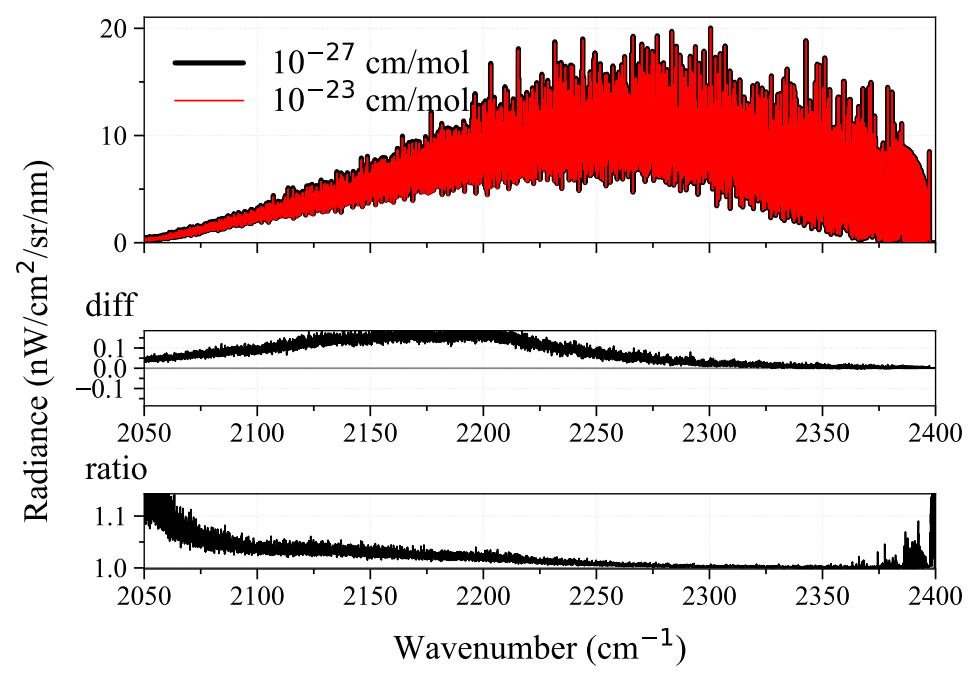

Figure 4: Effect of the linestrength cutoff $\left(10^{-23} \mathrm{~cm} /\right.$ molecule: red, $10^{-27} \mathrm{~cm} /$ molecule: black $)$ on the emission spectrum of the $4.2 \mu \mathrm{m}$ band of $\mathrm{CO}_{2}$ under the following conditions: $\mathrm{T}=3409 \mathrm{~K}, \mathrm{p}=1 \mathrm{~atm}, \mathrm{x}_{\mathrm{CO} 2}$ $=6.5 \times 10^{-5}, \mathrm{~L}=0.05 \mathrm{~cm}$, broadening cutoff: $50 \mathrm{~cm}^{-1}$, HITEMP-2010 database

2010, and those above $2200 \mathrm{~K}$ with CDSD-4000. Depending on the cutoff used, calculations with HITEMP-2010 are typically one to two orders of magnitude faster than with CDSD4000 . 


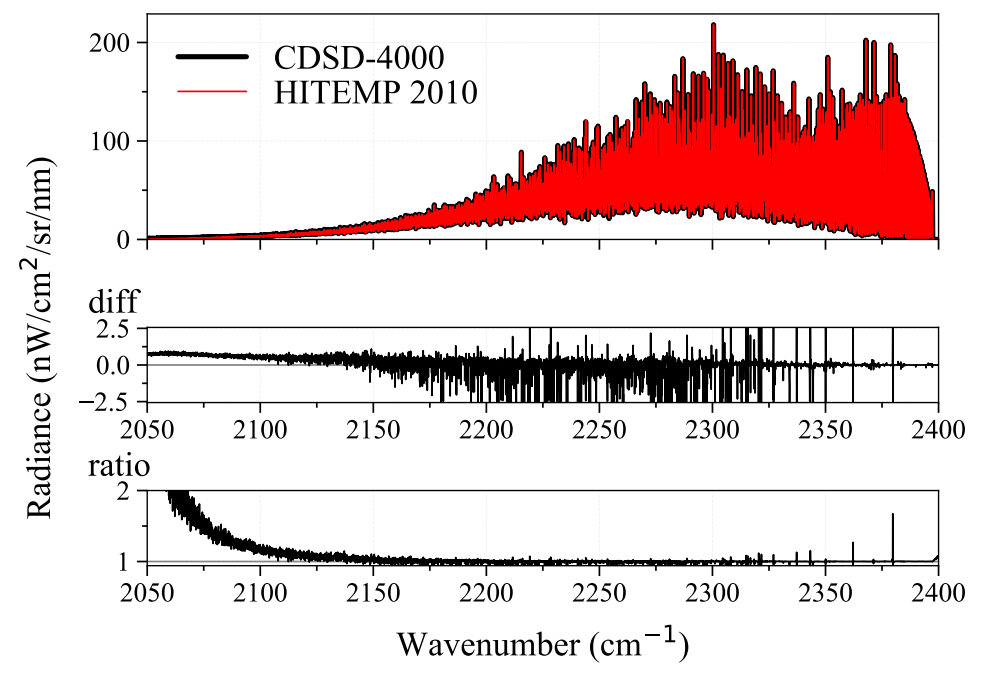

Figure 5: Influence of the line database (CDSD-4000: black and HITEMP-2010: red) on the emission spectrum of the $4.2 \mu \mathrm{m}$ band of $\mathrm{CO}_{2}$ under the following conditions: $\mathrm{T}=2200 \mathrm{~K}, \mathrm{p}=1$ atm, $\mathrm{x}_{\mathrm{CO}_{2}}=$ $3.3 \times 10^{-4}, \mathrm{~L}=0.05 \mathrm{~cm}$, broadening cutoff: $50 \mathrm{~cm}^{-1}$, linestrength cutoff: $10^{-27} \mathrm{~cm} / \mathrm{molecule}$

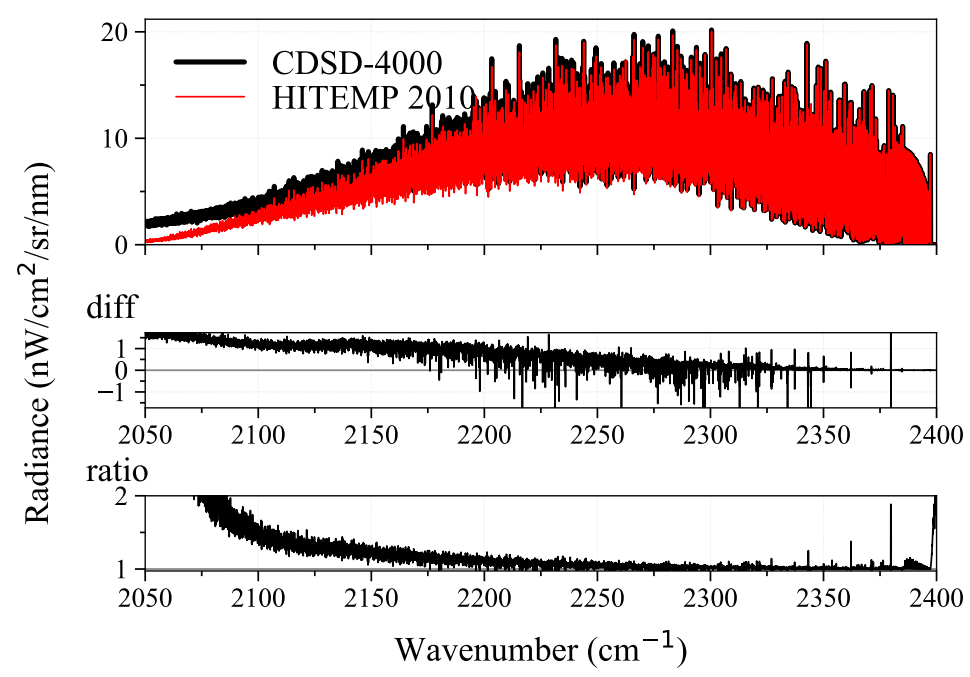

Figure 6: Influence of the line database (CDSD-4000: black and HITEMP-2010: red) on the emission spectrum of the $4.2 \mu \mathrm{m}$ band of $\mathrm{CO}_{2}$ under the following conditions: $\mathrm{T}=3409 \mathrm{~K}, \mathrm{p}=1$ atm, $\mathrm{x}_{\mathrm{CO}_{2}}=$ $6.5 \times 10^{-5}, \mathrm{~L}=0.05 \mathrm{~cm}$, broadening cutoff: $50 \mathrm{~cm}^{-1}$ (see section 3.4 ), linestrength cutoff: $10^{-27} \mathrm{~cm} / \mathrm{molecule}$

\subsection{Lineshape broadening cutoff}

For broadening calculations, the lineshape wings are cut beyond a certain range from the line center. The broadening cutoff has a strong impact on computation performance: because the Voigt lineshape in RADIS is calculated using the empirical approximation of Whiting [31], calculation times typically increase linearly with the number of spectral points (see the rule of thumb of the previous section), hence the broadening width cutoff has a strong 
impact on performances. However, at high pressure and temperatures, reducing the cutoff range can lead to significant error.

Figure 7 shows the effect of decreasing the cutoff range from 50 to $10 \mathrm{~cm}^{-1}$ on the $4.2 \mu \mathrm{m}$ emission band of $\mathrm{CO}_{2}$. Spectra are calculated with the HITEMP-2010 database for a pressure of $1 \mathrm{~atm}$, a $\mathrm{CO}_{2}$ mole fraction of $3.3 \times 10^{-4}$ and a path length of $0.05 \mathrm{~cm}$. The relative change in total radiance is about $1 \%$ in the band wings $\left(<2350 \mathrm{~cm}^{-1}\right)$, and reaches at most $3 \%$ in the bandhead region $\left(>2350 \mathrm{~cm}^{-1}\right)$. Thus, for pressure and temperature conditions similar to Fig. 7, the broadening range can be reduced to $10 \mathrm{~cm}^{-1}$ with minor impact on the results, and this value is used by default in RADIS.

The lineshape broadening can also create artifacts on the sides of the spectral range: to prevent that, RADIS automatically includes the contribution of out-of-range lines whose position is within the broadening width cutoff range.

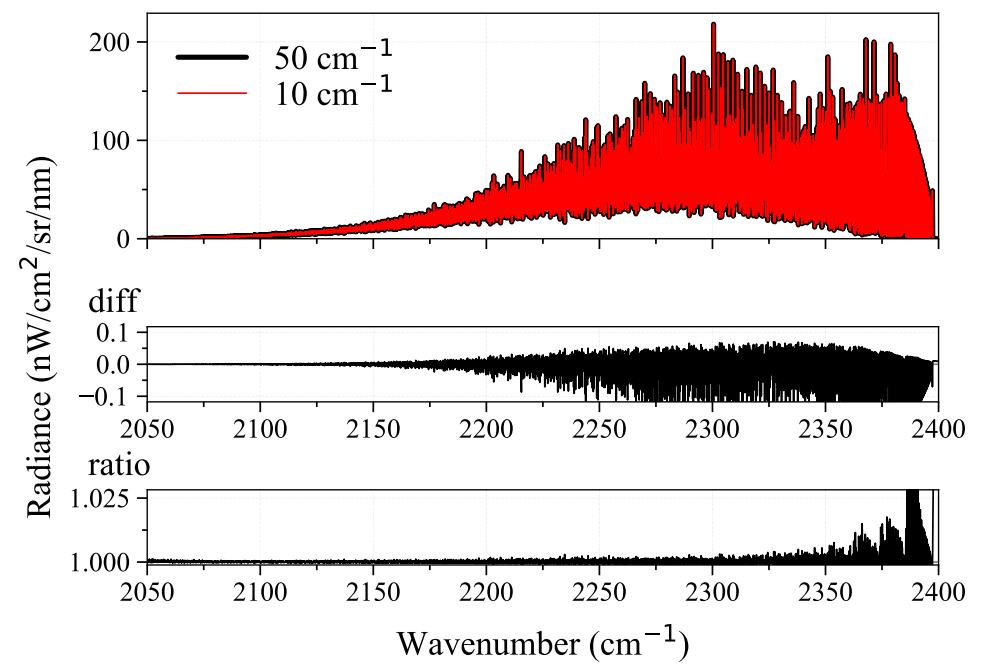

Figure 7: Effect of the line broadening cutoff range $\left(10 \mathrm{~cm}^{-1}\right.$ : red, $50 \mathrm{~cm}^{-1}$ : black $)$ on the emission spectrum of the $4.2 \mu \mathrm{m}$ band of $\mathrm{CO}_{2}$ under the following conditions: $\mathrm{T}=2200 \mathrm{~K}, \mathrm{p}=1 \mathrm{~atm}, \mathrm{x}_{\mathrm{CO}_{2}}=3.3 \times 10^{-4}$, $\mathrm{L}=0.05 \mathrm{~cm}$, linestrength cutoff $=10^{-27} \mathrm{~cm} /$ molecule, HITEMP-2010 database

\subsection{Pseudo-continuum, weak and strong lines}

Following the approach of other LBL codes [43, 10], a pseudo-continuum feature is implemented in RADIS, which reduces computation times by orders of magnitude while still allowing to capture the main features of a spectrum. NEQAIR [10] uses a tabulated pseudocontinuum for $\mathrm{CO}_{2}$ emission: only about $1 \%$ of the lines are fully resolved, i.e. their Voigt profile is calculated. NEQAIR pseudo-continuum are tabulated for different temperatures and narrow spectral ranges. In SPARTAN [20], lines are categorized at runtime as strong or weak depending on whether they are above or below a certain intensity threshold. The 
weak lines close to a strong line are not resolved. They are included in a pseudo continuum by adding their intensity to the nearest spectral point. Only about $10 \%$ of the lines are fully resolved in SPARTAN.

The pseudo-continuum in RADIS is similar to the one in SPARTAN, with weak lines categorized at runtime, but the categorization criterion is slightly different and weak lines are approximated with a rectangular lineshape.

The calculation procedure is as follows. First, an approximate spectral absorption coefficient $\widetilde{k}_{\lambda}$ is calculated by using a rectangular profile approximation for all lines. For a transition between two states $\eta$ and $\eta^{\prime}$, the Voigt profile Full-Width at Half-Maximum (FWHM) $\mathrm{w}_{\mathrm{v}}$ is computed using the empirical expression of Olivero and Longbothum [32]. An average spectral absorption coefficient $\widetilde{k}_{\lambda, \eta \eta^{\prime}}$ for this transition is calculated using a rectangular profile of width $\mathrm{w}_{\mathrm{v}}$. The approximate spectral absorption coefficient $\widetilde{k}_{\lambda}$ is calculated by summing the contribution of all lines. Lines are then labeled as weak if their average spectral absorption coefficient is much lower than the approximate spectral absorption coefficient:

$$
\widetilde{k}_{\lambda, \eta \eta^{\prime}}<\alpha \cdot \widetilde{k_{\lambda}}
$$

where the weak-line threshold $\alpha$ is user-defined, typically 0.01 . Lines that do not satisfy this criterion are labeled as strong lines. For strong lines, the spectral absorption coefficient $k_{\lambda}$ is calculated with a Voigt profile. Weak lines are aggregated in a pseudo-continuum by adding their average spectral absorption coefficient $\widetilde{k}_{\lambda, \eta \eta^{\prime}}$ over a rectangular lineshape of FWHM $\mathrm{w}_{\mathrm{v}}$. The pseudo-continuum is finally added to the spectral absorption coefficient $k_{\lambda}$ calculated from the strong lines.

For a $\mathrm{CO}_{2}$ spectrum in RADIS, a weak line threshold of 0.01 typically assigns $90 \%$ of the lines to the pseudo-continuum, and the overall computation efficiency is increased by an order of magnitude. Because weak lines are assigned to a continuum and not discarded, energyconservation is ensured. Because weak lines are assigned relative to the whole spectrum, features in the less intense parts of the spectrum are still conserved, which is not possible with an absolute linestrength cutoff such as the one used in section 3.2. Finally, because the assignation of weak lines is done at runtime, the method does not require any tabulated data, and is not restricted to specific thermo-chemical conditions nor limited to a given molecule.

Figure 8 shows a comparison of a $\mathrm{CO}_{2}$ emission spectrum calculated by resolving all lines (black) and by reducing the database with a pseudo-continuum calculation. The computational conditions are extreme, with a high temperature $(3409 \mathrm{~K})$, broad spectral range, low linestrength cutoff $\left(10^{-27} \mathrm{~cm} /\right.$ molecule), and large broadening width $\left(50 \mathrm{~cm}^{-1}\right)$. In the first case, more than $4 \times 10^{7}$ lines are resolved, for a total calculation time of 17 hours on a single CPU core. By using the pseudo continuum approach, with a threshold of 0.01 , the number of lines is reduced by a factor $190\left(2.1 \times 10^{5}\right.$ lines $)$ and the computation time is reduced 
to $1.3 \mathrm{hr}$. Fig. 8 shows that the spectral features are correctly reproduced. The agreement is excellent in the band head region $\left(>2350 \mathrm{~cm}^{-1}\right)$ where the spectrum is dominated by strong lines, and the error increases up to $10 \%$ in the hot band regions where the spectrum is a combination of numerous lines of similar amplitude. This error depends on the criterion (Eqn. 3.1) used for weak line assignation. Note that the criterion may lead to large errors if the number of overlapping strong lines of same intensity becomes close to $1 / \alpha$. We recommend using values below 0.01 (thus corresponding to a maximum of 100 potentially overlapping strong lines).

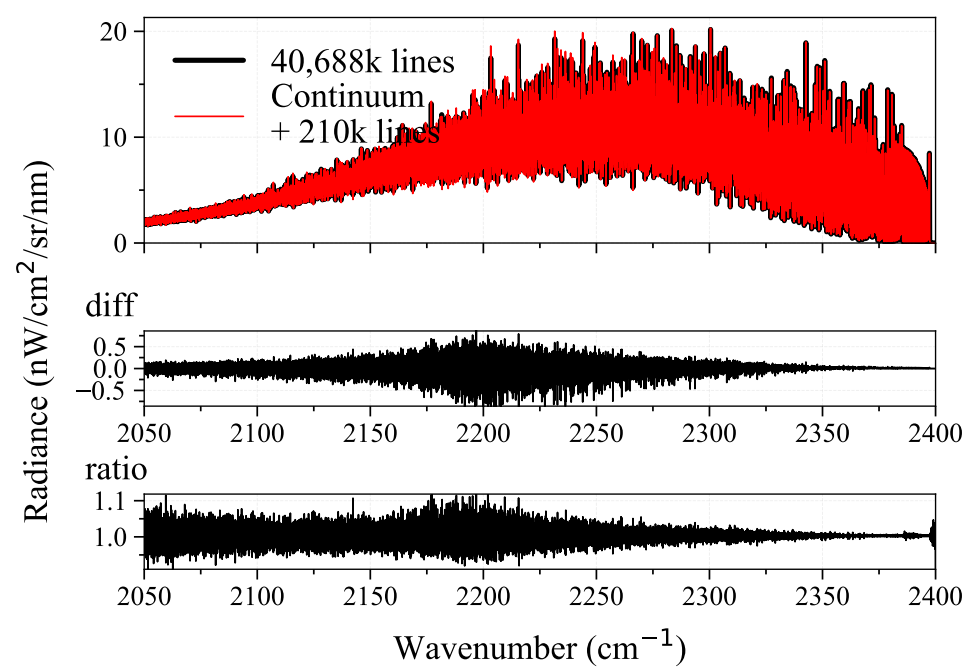

Figure 8: Calculation of the emission spectrum of the $4.2 \mu \mathrm{m}$ band of $\mathrm{CO}_{2}$ with all lines (black) and a reduced set of lines + continuum (weak line threshold of 0.01: red), under the following conditions: $\mathrm{T}=3409 \mathrm{~K}, \mathrm{p}$ $=1 \mathrm{~atm}, \mathrm{x}_{\mathrm{CO}_{2}}=6.5 \times 10^{-5}, \mathrm{~L}=0.05 \mathrm{~cm}$, linestrength cutoff $=10^{-27} \mathrm{~cm} /$ molecule, CDSD-4000 database

\subsection{Benchmark}

Performance numbers are given for a hardware configuration of 16 Gb RAM and an Intel i76700HQ 2.6 GHz CPU used on a single core. Without any optimization, RADIS calculations have performances comparable with Fortran codes. For instance, the nonequilibrium CO spectra of the validation case of Fig. 12 were calculated in $0.2 \mathrm{~s}$ in both RADIS and the Fortran code Specair. By combining the different optimization strategies presented in the previous sections, performances are further optimized.

As a first example, Figure 9 reproduces one of the benchmark cases of the HAPI code [5]: the absorption spectrum of methane is calculated at low temperature $(296 \mathrm{~K})$ and atmospheric pressure for all isotopes of the HITRAN database [44] from 0.001 to $11,500 \mathrm{~cm}^{-1}$. The database contains 450k lines. Similar computation parameters are used in both codes: the wavenumber resolution is $0.01 \mathrm{~cm}^{-1}$, and the RADIS broadening cutoff is $7 \mathrm{~cm}^{-1}$ which corresponds to about 50 times the HWHM of all lines, the parameter used in HAPI. The 
absorption coefficient calculation took 161 s in HAPI, $51 \mathrm{~s}$ in RADIS when resolving all lines, and only $2 \mathrm{~s}$ in the optimized case by applying a linestrength cutoff of $10^{-23} \mathrm{~cm} / \mathrm{molecule}$.

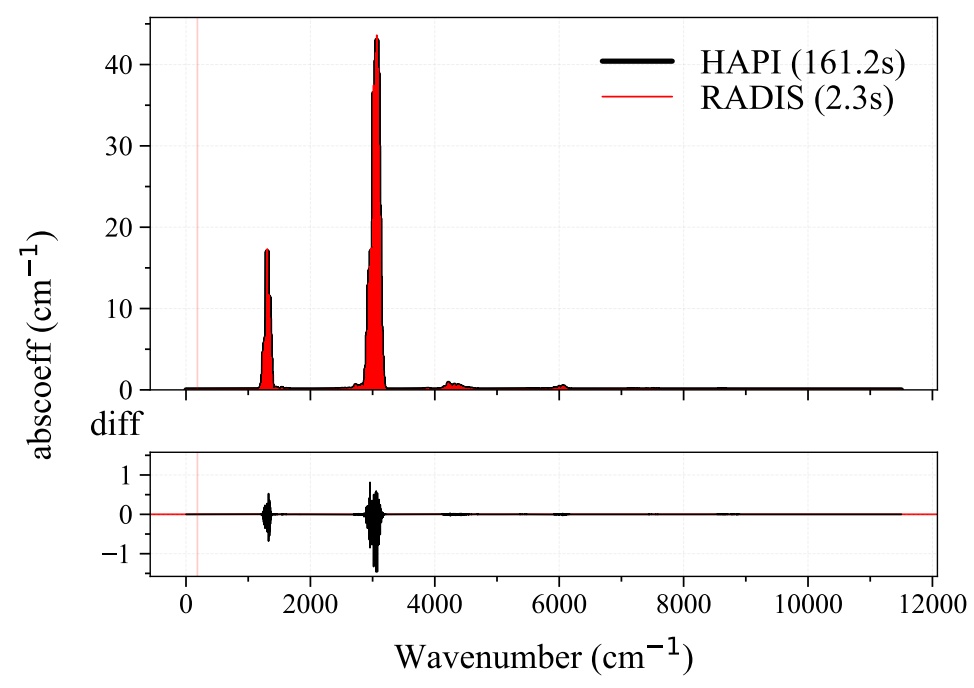

Figure 9: Spectral absorption coefficient of $\mathrm{CH}_{4}$ at $\mathrm{T}=300 \mathrm{~K}, \mathrm{p}=1 \mathrm{~atm}$ calculated with RADIS (red) and HAPI [5] (black) from the HITRAN-2016 [44] database (450k lines). Resolving all lines took $161 \mathrm{~s}$ in HAPI and $51 \mathrm{~s}$ in RADIS. An optimized spectrum (16k lines) is calculated in $2 \mathrm{~s}$ in RADIS (linestrength cutoff $=$ $10^{-23} \mathrm{~cm} /$ molecule).

Similarly, Figure 10 shows a $\mathrm{H}_{2} \mathrm{O}$ transmittance spectrum calculated at high temperature (2200 K) using RADIS and the HITEMP-2010 [26] database. The reference case (black) contains $3 \mathrm{M}$ lines, and requires a calculation time of $40 \mathrm{~min}$. In the optimized case, the calculation times are reduced down to $25 \mathrm{~s}$ with an error of less than $0.1 \%$.

\section{Multi-slab module}

A slab of length $\mathrm{L}$ is defined as a homogeneous section of gas of uniform spectral emission density $\mathrm{j}_{\lambda}\left(\mathrm{W} / \mathrm{cm}^{3} / \mathrm{sr} / \mathrm{nm}\right)$, absorption coefficient $\mathrm{k}_{\lambda}\left(\mathrm{cm}^{-1}\right)$ and transmittance $\tau_{\lambda}$. The spectral radiance $\mathrm{I}_{\lambda}\left(\mathrm{W} / \mathrm{cm}^{2} / \mathrm{sr} / \mathrm{nm}\right)$ is computed by solving the radiative transfer equation analytically, neglecting scattering processes.

$$
I_{\lambda}=j_{\lambda} \frac{\left(1-\exp \left(-k_{\lambda} L\right)\right)}{k_{\lambda}}
$$

In the multi-slab model, two precomputed slabs $\mathrm{S}_{1}$ and $\mathrm{S}_{2}$ can be added along the lineof-sight: the resulting slab $\left(\mathrm{S}_{1} \rightarrow \mathrm{S}_{2}\right)$ is calculated from the spectral radiance $\mathrm{I}_{\lambda 1}, \mathrm{I}_{\lambda 2}$ and spectral transmittance $\tau_{\lambda 1}, \tau_{\lambda 2}$ of the two slabs as:

$$
\left(\begin{array}{c}
I_{\lambda 1} \\
\tau_{\lambda 1}
\end{array}\right) \rightarrow\left(\begin{array}{c}
I_{\lambda 2} \\
\tau_{\lambda 2}
\end{array}\right)=\left(\begin{array}{c}
I_{\lambda 1} \tau_{\lambda 2}+I_{\lambda 2} \\
\tau_{\lambda 1} \tau_{\lambda 2}
\end{array}\right)
$$




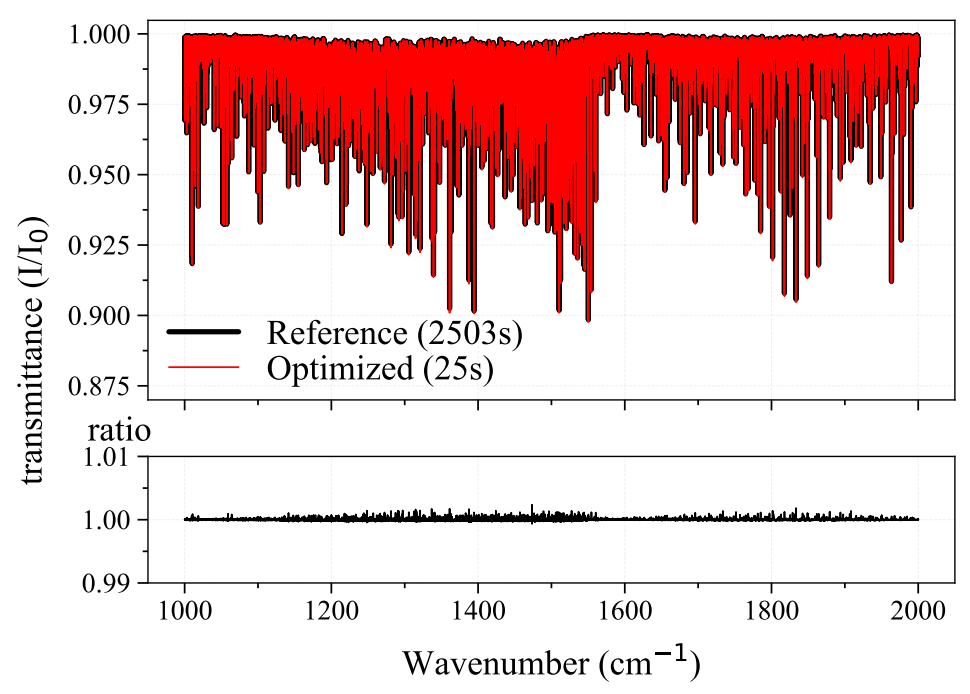

Figure 10: Broad-band $\mathrm{H}_{2} \mathrm{O}$ transmittance spectrum at $\mathrm{T}=2200 \mathrm{~K}, \mathrm{p}=1 \mathrm{~atm}, \mathrm{x}_{\mathrm{H}_{2} \mathrm{O}}=0.1$, calculated with the HITEMP-2010 [26] database. In the reference case (black), 3M lines are calculated (linestrength cutoff $=10^{-27} \mathrm{~cm} /$ molecule, broadening cutoff $=30 \mathrm{~cm}^{-1}$ ). The optimized version (red) resolves $114 \mathrm{k}$ lines and adds $213 \mathrm{k}$ lines in a pseudo-continuum (linestrength cutoff $=10^{-25} \mathrm{~cm} /$ molecule, broadening cutoff $=$ $\left.5 \mathrm{~cm}^{-1}\right)$

The spectral contribution of different species occupying the same volume can also be computed separately and then merged, i.e. the emission and absorption of different species are added. The resulting merged slab $\mathrm{S}_{1}+\mathrm{S}_{2}$ is calculated from the spectral emission density $\mathrm{j}_{\lambda 1}, \mathrm{j}_{\lambda 2}$ and spectral absorption coefficients $\mathrm{k}_{\lambda 1}, \mathrm{k}_{\lambda 2}$ of the initial slabs as:

$$
\left(\begin{array}{l}
j_{\lambda 1} \\
k_{\lambda 1}
\end{array}\right)+\left(\begin{array}{l}
j_{\lambda 2} \\
k_{\lambda 2}
\end{array}\right)=\left(\begin{array}{l}
j_{\lambda 1}+j_{\lambda 2} \\
k_{\lambda 1}+k_{\lambda 2}
\end{array}\right)
$$

The radiative transfer equation is solved as in Eqn. 2.4 to derive the radiance and transmittance. The merged slab definition of Eqn. 4.3 is a first order approximation that is valid only if the lineshape $\Phi$ is not impacted by the change in gas composition. In the fitting process, however, it allows to change the gas composition by merging precomputed slabs of pure gases (ex: $\mathrm{CO}_{2}, \mathrm{CO}, \mathrm{H}_{2} \mathrm{O}$ ), which avoids recalculating the broadening of individual lines and yields considerable savings in computer time. If the collisional broadening coefficients are significantly modified by the change in gas composition, the emission and absorption spectra should be recomputed with the actual gas composition.

\section{Validation cases}

RADIS is validated by comparisons with other spectral codes and experimental results in local thermodynamic equilibrium (LTE) and nonequilibrium. These comparisons are used to successively ensure the correct implementation of line broadening, of the calculation of 
populations in nonequilibrium cases, and of the overall fitting of high temperature LTE spectra.

\subsection{Line broadening}

Figure 11 compares the simulations obtained with RADIS and with the HAPI [5] code. The HITRAN-2016 database is used in both cases to compute the transmittance spectrum of the main isotope of $\mathrm{CO}_{2}\left({ }^{12} \mathrm{C}^{16} \mathrm{O}^{16} \mathrm{O}\right)$ around $2286.90 \mathrm{~cm}^{-1}$, for a temperature of $\mathrm{T}=1500 \mathrm{~K}$, and a pressure of 100 mbar. Under these conditions, the FWHM of the Doppler and collisional broadening have the same order of magnitude, and the lineshape has a Voigt profile. The perfect agreement between the two simulations validates the spectral model under equilibrium conditions, including line broadening.

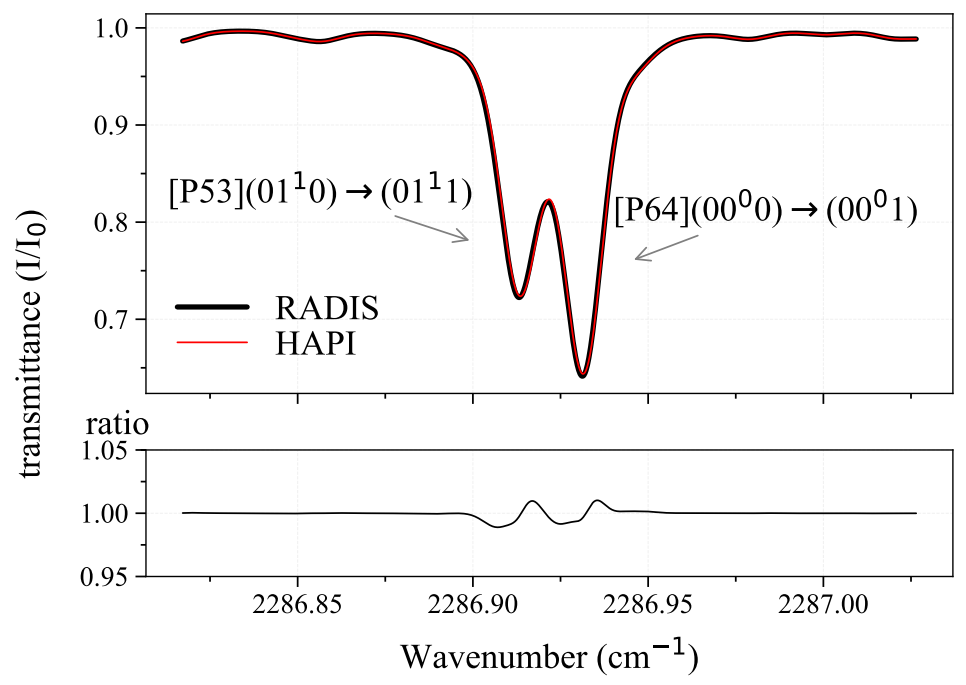

Figure 11: Comparison of RADIS and HAPI calculations of the transmittance of ${ }^{12} \mathrm{C}^{16} \mathrm{O}^{16} \mathrm{O}$ at $\mathrm{T}=1500 \mathrm{~K}$, $\mathrm{p}=0.1$ bar, $\mathrm{L}=0.1 \mathrm{~cm}, \mathrm{x}_{\mathrm{CO}_{2}}$

\subsection{Nonequilibrium, two-temperature spectra}

To ensure that the rovibrational energy partitioning into $\mathrm{E}_{\mathrm{rot}}^{\mathrm{i}}$ and $\mathrm{E}_{\mathrm{vib}}^{\mathrm{i}}$ is correct in RADIS, it is necessary to consider nonequilibrium validation cases. Figure 12 shows the absorption coefficient of the $\operatorname{CO}(\mathrm{X}, \Delta \mathrm{v}=+1)$ band obtained with Specair $[2,3]$ and RADIS for vacuum wavelengths from 4500 to $4900 \mathrm{~nm}$, under equilibrium (a) and nonequilibrium (b) conditions. In this example, the HITEMP 2010 [26] database is used to calculate line intensities in RADIS. Energies are calculated using the Dunham expansions mentioned in section 2.5.

Figure 12 shows excellent agreement in the magnitude and distribution of the absorption lines. However, small differences in the line intensities remain. The differences can be explained by differences in the rovibrational lineshapes calculated by both codes. In particular, 


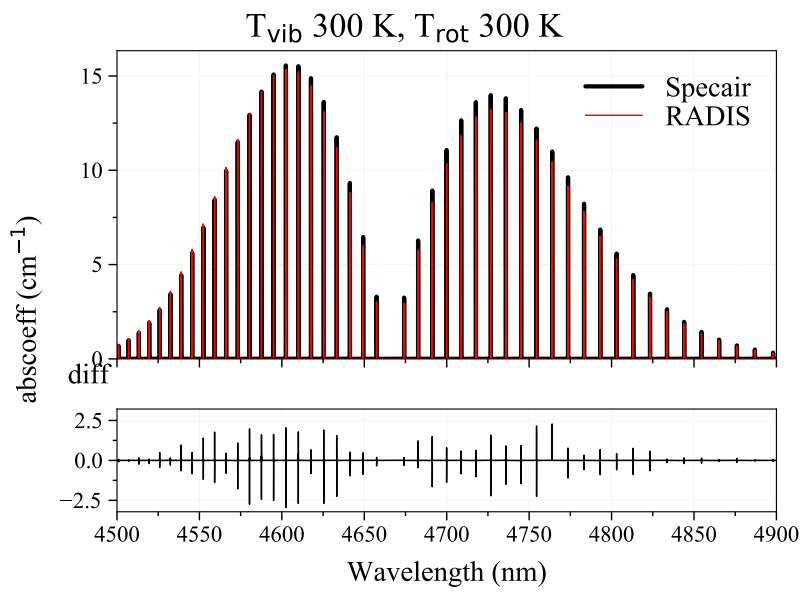

(a)

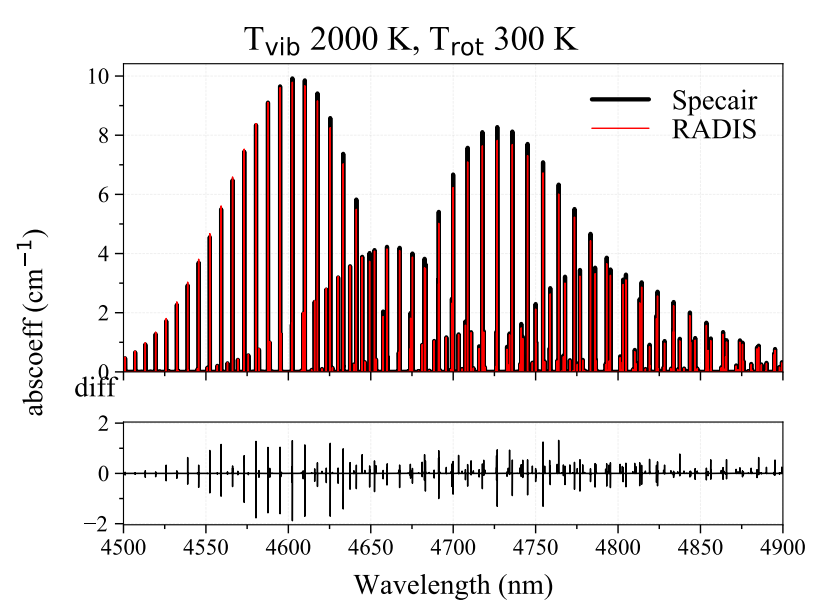

(b)

Figure 12: Nonequilibrium calculations of the absorption coefficient of the $\operatorname{CO}(\mathrm{X}, \Delta \mathrm{v}=+1)$ band with Specair (black) and RADIS (red), for low rotational temperatures. $\mathrm{P}=0.01$ bar, $\mathrm{x}_{\mathrm{CO}}=1$

the lineshapes in Specair appear thinner than the lineshapes in RADIS for the low rotational levels of the $\mathrm{R}$ branch near $4650 \mathrm{~nm}$, and become broader for the higher rotational levels near $4550 \mathrm{~nm}$. Specair uses Van der Waals and resonance broadening widths derived from Eqns. 10 and 11 of Ref. [45], which are constant for all lines of a given isotope, whereas RADIS uses the collisional broadening width given in the line database, which are rovibrational specific. It was verified that the differences between both codes are amplified in calculations at higher pressures, which suggest that the collisional broadening coefficients are slightly different.

Differences in the lineshapes would not change the absorption coefficient integrated over the lineshape. We compared the integral of the calculated spectra in both codes, and the difference was found to be up to $2.4 \%$ and $2.5 \%$ for the two cases of Fig. 12. This difference can be explained by the rovibrational Einstein coefficients used in the two spectral codes: Specair uses vibrational Einstein coefficients multiplied by Hönl-London factors, whereas RADIS uses the experimentally measured HITRAN rovibrational specific Einstein coefficients. In its current version (3.0), Specair does not use Herman-Wallis factors, which describe the effect of rotational-vibrational coupling on the line intensities. This interaction is negligible in rovibronic transitions where the energy separation between the upper and lower levels is large, but it can become significant for rovibrational transitions where the energy separation is less important.

Third, offsets of up to $0.01 \mathrm{~nm}$ can be observed between the line positions of the two spectral codes, which also result in differences in the amplitude. Therefore, we believe that the quantitative differences found in both codes can be attributed to differences in the line databases, and that the nonequilibrium populations are correctly calculated in RADIS.

We also compared nonequilibrium rovibrational partition functions obtained with RADIS 
and Specair, which resulted in a perfect agreement, and confirm the correct implementation of nonequilibrium partition functions in RADIS.

\subsection{Nonequilibrium, vibrational-mode-temperature spectra}

For $\mathrm{CO}_{2}$, RADIS can use several types of two-temperature Boltzmann or Treanor nonequilibrium distributions, but also Boltzmann and Treanor distributions with one temperature per vibrational mode. For such distributions, few reference cases exist. Dang et al. [34] performed diode-laser transmittance measurements in a continuous-wave laser discharge at 20 mbar with $10 \%$ of $\mathrm{CO}_{2}$ (in mass fractions). Klarenaar et al. [7] successfully reproduced these transmittance measurements using a 3-temperature nonequilibrium model, with a $\mathrm{T}_{\mathrm{vib}_{1,2},}, \mathrm{~T}_{\mathrm{vib}_{3}}$ Treanor vibrational distribution, and a $\mathrm{T}_{\text {rot }}$ rotational Boltzmann distribution. The translational temperature is assumed to be equal to the rotational temperature.

We use this transmittance spectrum as a validation case for multi-temperature nonequilibrium calculations in RADIS, using the same input conditions as Klarenaar et al. [7]. The line database is HITEMP-2010 [26], and the Dunham coefficients are the one given in Ref. [7]. However, we extend the nonequilibrium distribution to a 4-temperature distribution, with a $T_{\text {vib }_{1}}, T_{\text {vib }_{2}}, T_{\text {vib }_{3}}$ Treanor distribution for vibrational levels, and a $T_{\text {rot }}$ Boltzmann distribution for rotational levels. Figure 13 shows the output of a fitting algorithm where all temperatures were initially set to $\mathrm{T}=1000 \mathrm{~K}$. The best fit values are $\mathrm{T}_{\mathrm{vib}_{1}}=497 \mathrm{~K}, \mathrm{~T}_{\text {vib }_{2}}$ $=507 \mathrm{~K}, \mathrm{~T}_{\text {vib }_{3}}=2559 \mathrm{~K}, \mathrm{~T}_{\text {rot }}=482 \mathrm{~K}$. Two conclusions are obtained. First, the obtained temperatures are close to those obtained by Klarenaar et al. [7] $\left(\mathrm{T}_{\mathrm{vib} 1,2}=517 \mathrm{~K}, \mathrm{~T}_{\mathrm{vib} 3}=\right.$ $2641 \mathrm{~K}, \mathrm{~T}_{\mathrm{rot}}=491 \mathrm{~K}$ ), which validates multi-temperature non-equilibrium calculations in RADIS. Second, $\mathrm{T}_{\mathrm{vib}_{1}}$ is found to be approximately equal to $\mathrm{T}_{\mathrm{vib}_{2}}$, which confirms that the symmetric stretching and bending modes mode of $\mathrm{CO}_{2}$ are in equilibrium, and thus justify the use of a 3-temperature model in the work of Klarenaar et al. [7].

\subsection{Multi-slab model}

In this section, we validate the multi-slab module against line-of-sight calculations and experiments. As a reference, we use the high resolution $\mathrm{CO}_{2}$ FTIR spectra measured by Depraz et al. $[46,47]$ in an atmospheric pressure microwave plasma torch operated with pure CO2. The authors measured the temperature profile at the exit of a 40-mm diameter tube from the Abel-inverted emission of the second overtone of CO at $2.3-2.5 \mu \mathrm{m}$. The profile has a peak temperature of $3500 \mathrm{~K}$ at the center of the plasma and is extrapolated to the tube wall at $850 \mathrm{~K}$. Depraz et al. compared their measurements with simulations obtained with their LBL code and two spectral databases (HITELOR and CDSD-4000), and obtained excellent agreement with CDSD-4000.

To validate the line-of-sight model, we compare RADIS with the LBL code of Depraz et al. 


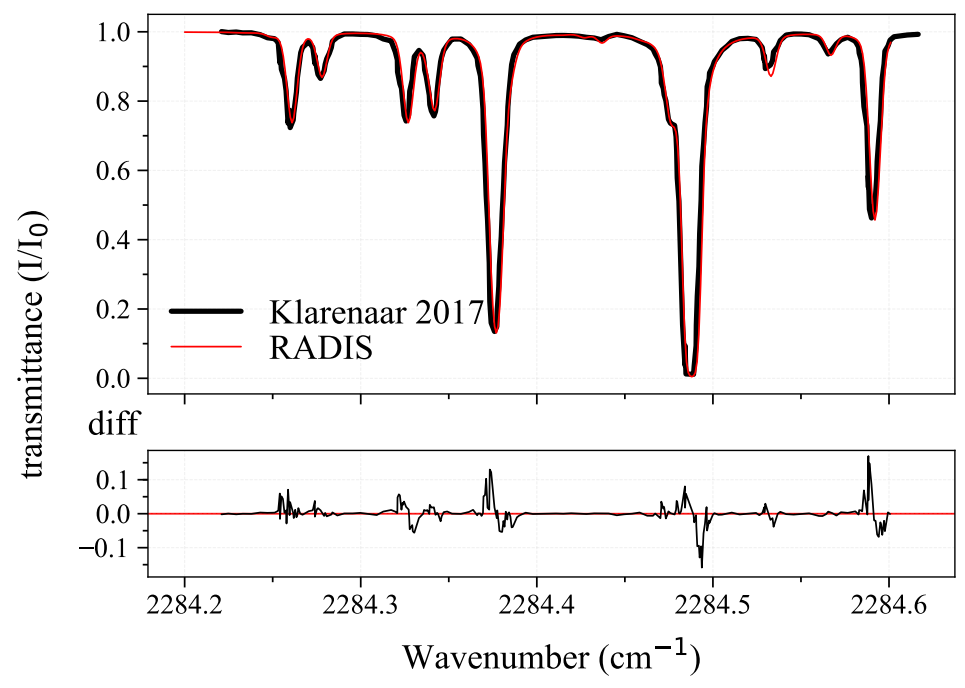

Figure 13: Nonequilibrium $\mathrm{CO}_{2}$ transmittance spectrum, calculated by Klarenaar et al. [7] from the data of Dang et al. [34] (black), is fitted in RADIS using a 4-temperature Treanor distribution: $\mathrm{T}_{\mathrm{vib}_{1}}=497 \mathrm{~K}$, $\mathrm{T}_{\mathrm{vib}_{2}}=507 \mathrm{~K}, \mathrm{~T}_{\mathrm{vib}_{3}}=2559 \mathrm{~K}, \mathrm{~T}_{\text {trans }}=\mathrm{T}_{\text {rot }}=482 \mathrm{~K}(\mathrm{red})$. Experimental conditions: $\mathrm{p}=20$ mbar, $10 \%$ $\mathrm{CO}_{2}$ (mass fraction)

We use the same line database (CDSD-4000) and the same broadening range of $50 \mathrm{~cm}^{-1}$. To our knowledge, the calculations performed in Depraz et al. [46] did not specify a linestrength cutoff. In our simulations, we used a cutoff of $10^{-27} \mathrm{~cm} /$ molecule, which has been shown in section 3.2 (Figure 2) to be sufficient for the temperatures of interest here (up to $3500 \mathrm{~K}$ ). Simulations were performed with the temperature profile measured by Depraz et al. [46] $20 \mathrm{~mm}$ above the plasma torch exit. The profile was divided into 41 homogeneous slabs, as shown in Figure 14. The gas composition was taken as the equilibrium composition at the local temperature and atmospheric pressure.

Figure 15 shows a comparison of the $\mathrm{CO}_{2}$ emission spectra in the $3758-3764.5 \mathrm{~cm}^{-1}$ spectral range calculated with RADIS and by Depraz et al., both using the CDSD-4000 database. An instrumental broadening of $F W H M=0.016 \mathrm{~cm}^{-1}$ with a triangular shape is applied. The excellent agreement validates the RADIS simulations and the radiative transfer equation solver in the multi-slab configuration. The differences fall within the error margin due to the discretization of the temperature profile, and the uncertainty on the cutoff criteria used by the Depraz et al..

In Figure 16, we compare our simulated spectrum with the experimental spectrum obtained by Depraz et al. [46], this time by looking at the bandhead of $\mathrm{CO}_{2}$ at $4.2 \mu \mathrm{m}$. The author already observed a good agreement between CDSD-4000 and their measurements, and the present calculation is just a further validation of the line-of-sight capabilities of RADIS. 


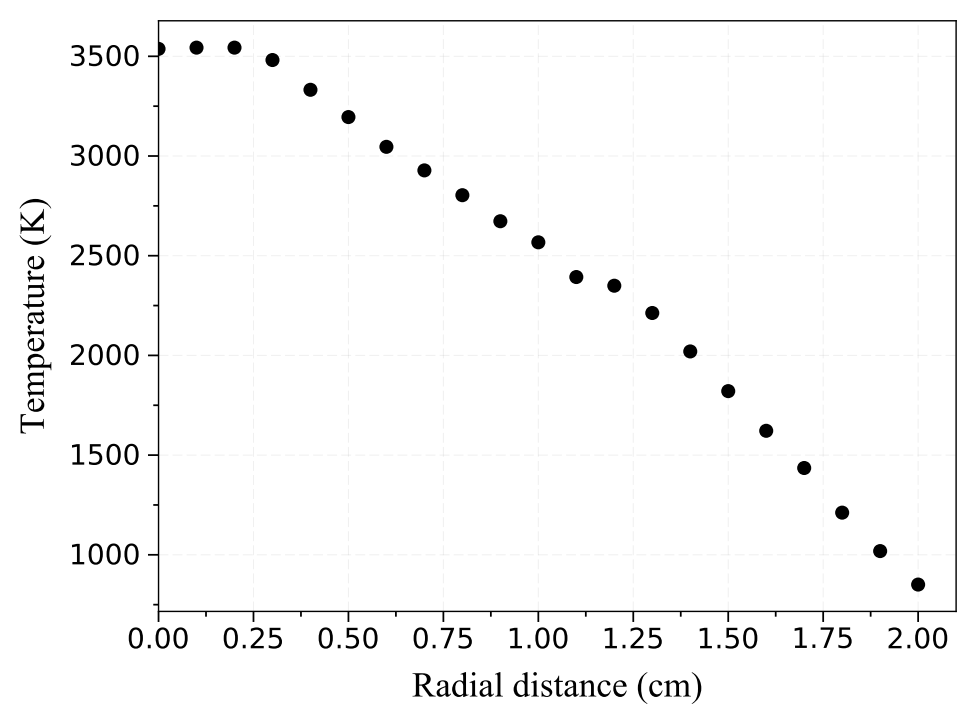

Figure 14: Temperature half-profile discretized from Depraz et al. [46], used as input for our validation case. The mole fraction profiles are determined from this temperature profile, assuming LTE at $\mathrm{P}=1 \mathrm{~atm}$.

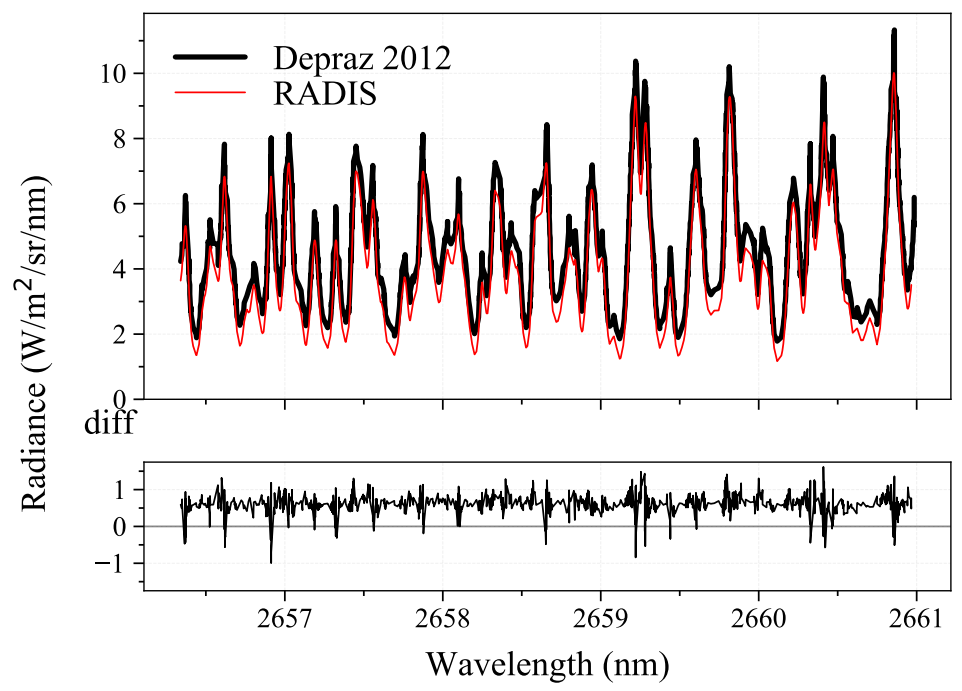

Figure 15: Simulated line-of-sight emission spectrum of a microwave-torch plasma (pure $\mathrm{CO}_{2}, 1$ atm), compared with simulations from Depraz et al. [46]. RADIS simulations use the CDSD-4000 database. The radiative transport equation is solved along a line-of-sight comprised of 41 slabs (see Fig. 14), and the final spectrum is convolved with a triangular slit function of $\mathrm{FWHM}=0.016 \mathrm{~cm}^{-1}$.

\subsection{Comparison with EAST shock-tube experiment}

RADIS can be used to model radiation from high-temperature shock-tube experiments. As a validation case, we reproduce the radiation of the $4.2 \mu \mathrm{m} \mathrm{CO}_{2}$ band measured by Cruden et al. [48] in the NASA EAST facility, for a $3.12 \mathrm{~km} / \mathrm{s}$ shock produced in a $1.33 \mathrm{mbar}$, $300 \mathrm{~K}$ pure $\mathrm{CO}_{2}$ gas. The CEA code [49] is used to calculate the post-shock conditions and 


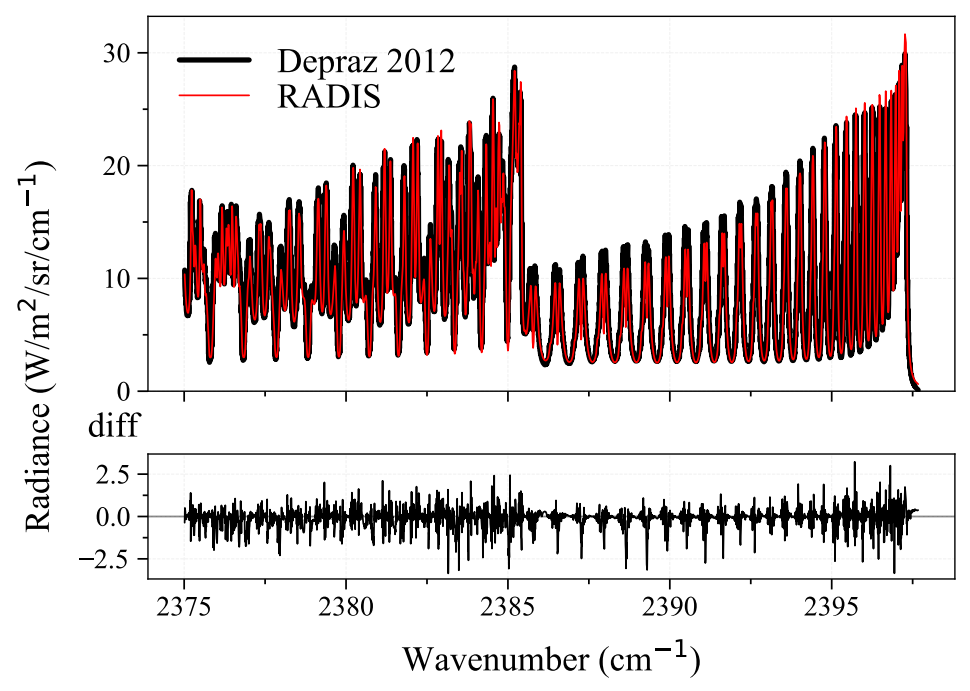

Figure 16: Simulated line-of-sight emission spectra of a microwave-torch plasma (pure $\mathrm{CO}_{2}, 1$ atm), plasma, compared with experimental data from Depraz et al. [46]. The RADIS calculation uses CDSD-4000 with a line-of-sight of 41 slabs convolved with a triangular slit function of $\mathrm{FWHM}=0.016 \mathrm{~cm}^{-1}$

yields $\mathrm{T}=3967 \mathrm{~K}, \mathrm{p}=220$ mbar and $\mathrm{x}_{\mathrm{CO}_{2}}=1$ if the chemistry is considered frozen during the shock, and $\mathrm{T}=2611 \mathrm{~K}, \mathrm{p}=220 \mathrm{mbar}, \mathrm{x}_{\mathrm{CO}_{2}}=0.62$ if the gas has relaxed to equilibrium. Fig. 17 shows the spectra calculated with RADIS under both conditions and using the CDSD-4000 database. The frozen-state calculation matches the experimental data, in perfect agreement with the conclusions of Cruden et al. [48] using the NEQAIR [9] code.

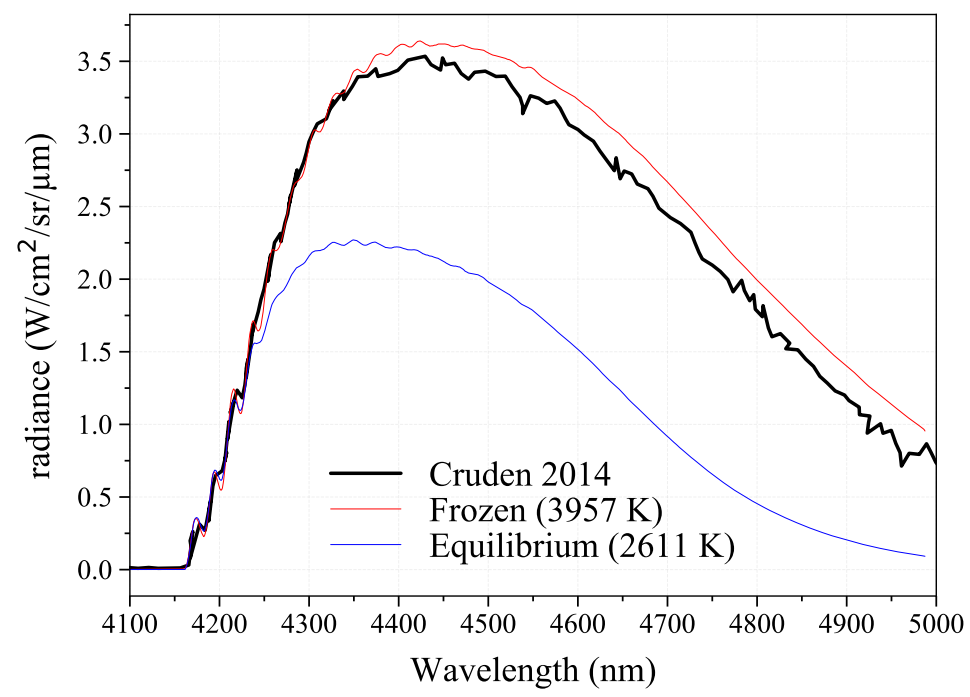

Figure 17: $\mathrm{CO}_{2}$ emission spectrum of a $3.12 \mathrm{~km} / \mathrm{s}$ shock-tube experiment (black [48]), compared with calculations in a frozen state ( $\mathrm{red}: \mathrm{T}=3967 \mathrm{~K}, \mathrm{x}_{\mathrm{CO}_{2}}=1, \mathrm{p}=220 \mathrm{mbar}$ ) and equilibrium state (blue: $\mathrm{T}=2611 \mathrm{~K}$, $\mathrm{x}_{\mathrm{CO}_{2}}=0.62, \mathrm{p}=220$ mbar). Calculation parameters: $\mathrm{L}=10.16 \mathrm{~cm}, \mathrm{CDSD}-4000$ database. 


\subsection{Modeling of spectra obtained in an atmospheric pressure RF torch plasma}

RADIS is finally applied to improve the current modeling of the experimental emission spectra obtained in an equilibrium RF plasma [25] torch.

Packan et al. [25] measured the absolute-intensity $\mathrm{CO}_{2}$ spectrum emitted by an air plasma produced by a radio-frequency plasma torch. In the year the experiments were conducted (1997), the $\mathrm{CO}_{2}$ concentration in atmospheric air was $363 \mathrm{ppm}$. The plasma was shown to be in LTE, and a temperature profile was determined from the absolute intensity of the NO bandhead at $5.1 \mu \mathrm{m}$ [25]. The peak temperature at the plasma center was $3400 \mathrm{~K}$. Packan et al. [25] compared their measurements with simulations of $\mathrm{CO}_{2}$ emission by means of the correlated-k model (c-k) of Pierrot et al. [50]. The simulations were found to overpredict the measurements by about $50 \%$, likely because the c-k model was used at temperatures slightly above its domain of validity.

To simulate the torch spectrum in RADIS, we use a spatial model containing 23 slabs for the plasma and one slab for the 6-m of atmospheric air along the optical path from the plasma to the $\mathrm{CCD}$ camera. The concentrations of $\mathrm{CO}_{2}$ and $\mathrm{CO}$ are calculated assuming LTE. The emission intensity per $\mathrm{CO}_{2}$ molecule increases with the temperature, but the number density of $\mathrm{CO}_{2}$ molecules decreases under the effect of dissociation. Thus, there is a peak of emission at about $2400 \mathrm{~K}$ for the $4.2 \mu \mathrm{m}$ band of $\mathrm{CO}_{2}$. Figure 18 shows the radial temperature profile in the plasma, measured out to a radius of $0.55 \mathrm{~cm}$ (note that the measurements are performed $1 \mathrm{~cm}$ downstream of the torch exit nozzle which had a radius of $0.5 \mathrm{~cm}$ ). Because the plasma exits the test-section at high velocity (about $400 \mathrm{~m} / \mathrm{s}$ ), the temperature should fall off rapidly beyond a radius of $0.5 \mathrm{~cm}$. Thus, we extrapolate the measured profile by assuming that the temperature decreases to $300 \mathrm{~K}$ at a radius of $0.6 \mathrm{~cm}$. Figure 18 also shows the radial emission intensity profile of the CO2 $4.2 \mu \mathrm{m}$ band. Most of the emission comes from the slabs at outer radii where the temperature is around $2400 \mathrm{~K}$.

We apply an instrumental slit function with a trapezoidal shape based on the characteristics of the monochromator used by Packan et al. [25]: the entrance and exit slits are 1 and $2.8 \mathrm{~mm}$, and the reciprocal linear dispersion is approximately $4 \mathrm{~nm} / \mathrm{mm}$. The experimental spectrum in Ref. [25] is given in $\mu \mathrm{W} / \mathrm{cm}^{2} / \mathrm{sr}$, which corresponds to an instrumental slit function normalized to an amplitude equal to 1 instead of the area equal to 1 commonly found in the literature. The measured intensity is converted to $\mu \mathrm{W} / \mathrm{cm}^{2} / \mathrm{sr} / \mathrm{nm}$ by dividing by the equivalent slit function FWHM (11.2 nm here).

Figure 19 (red) compares the experimental spectrum of [25] with the spectrum computed with RADIS (CDSD-4000 for $\mathrm{T} \geq 2200 \mathrm{~K}$, and HITEMP-2010 for $\mathrm{T} \leq 2200 \mathrm{~K}$ ) using the temperature profile of Fig. 18. CO radiation(blue line) is included in the calculation but contributes less than $10 \%$ to the measured emission (blue line). The RADIS simulation is compared with the c-k model simulation presented in [25], scaled to a concentration of $363 \mathrm{ppm}$ assuming an optically thin plasma (black squares). The RADIS spectrum repro- 


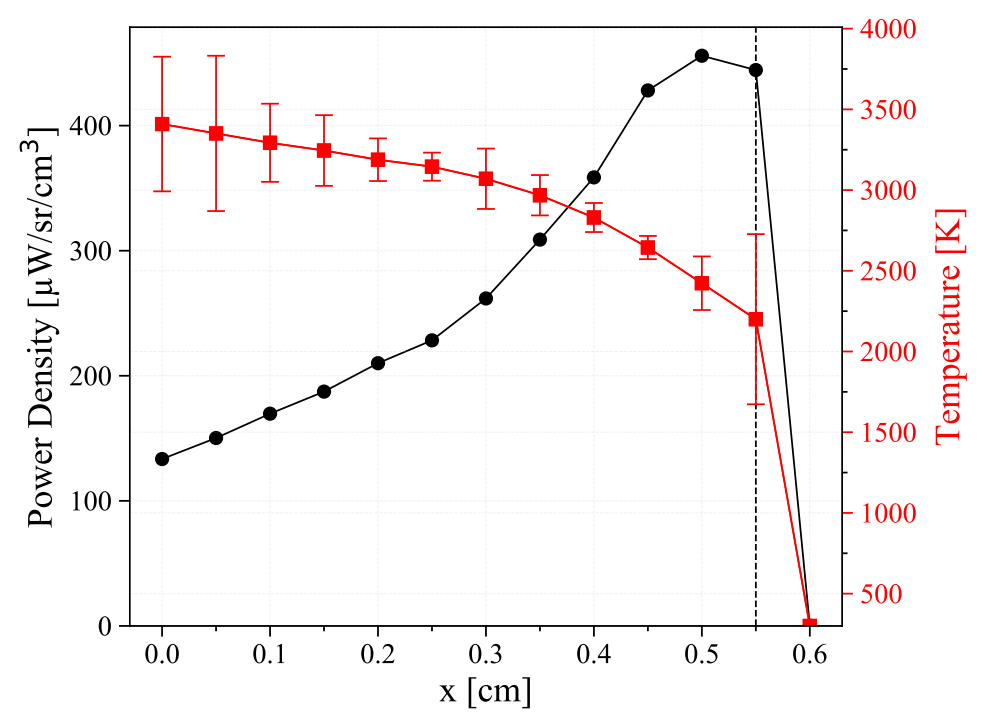

Figure 18: Radial temperature profile in the plasma produced by the RF torch, as measured by Packan et al. [25] (red), and power density of the $\mathrm{CO}_{2} 4.2 \mu \mathrm{m}$ band assuming chemical equilibrium in the plasma initially composed of air with $363 \mathrm{ppm}$ of $\mathrm{CO}_{2}$ (black)

duces the overall shape and absolute intensity slightly better than the c-k model. However, large differences with the experimental spectrum are observed in the $4.2-4.2 \mu \mathrm{m}$ and the $4.4-4.7 \mu \mathrm{m}$ regions. Both deviations are explained and corrected below.

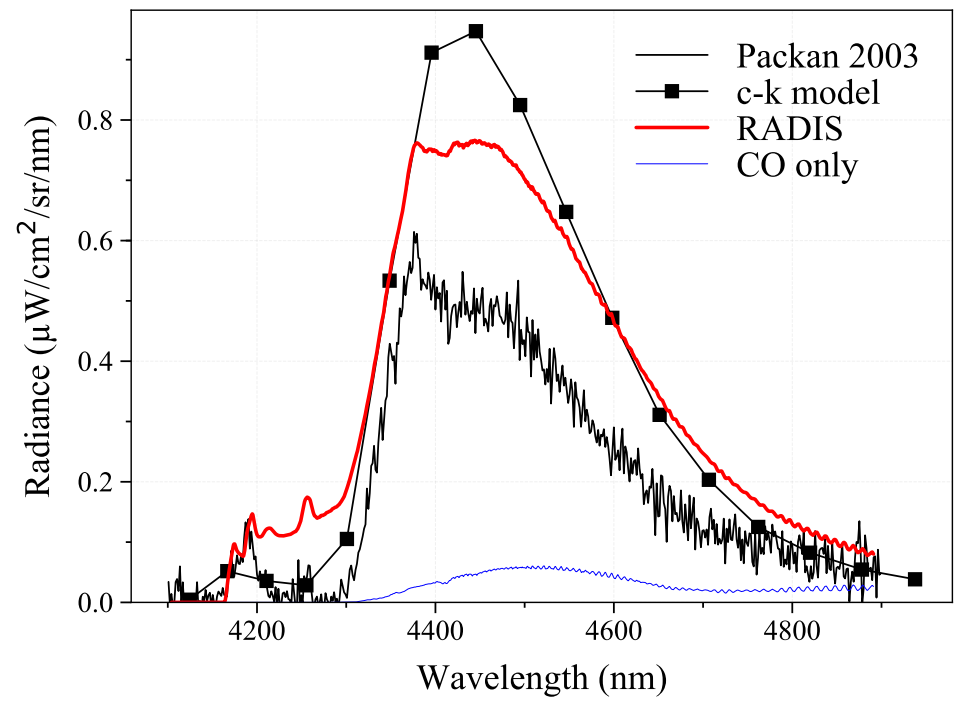

Figure 19: Simulation of an LTE plasma: experimental data (black, [25]), total emission along the line-ofsight (11 slabs $+6 \mathrm{~m}$ air) simulated with the c-k model scaled to $363 \mathrm{ppm}$ (black) and with RADIS (red). The contribution of $\mathrm{CO}$ to the total emission is also shown (blue).

The discrepancy in the $4.2-4.3 \mu \mathrm{m}$ region, where absorption by ambient $\mathrm{CO}_{2}$ is important, can be explained by carefully considering the experimental procedure used in Ref. [25]: 
the spectrum was acquired with a lock-in amplifier, using a chopper placed right at the spectrometer entrance. Consequently, although the light emitted by the plasma travels through 6 meters of atmospheric air, the emission of the atmospheric $\mathrm{CO}_{2}$ at $300 \mathrm{~K}$ present along the optical path inside the spectrometer (about 3 meters) is experimentally recorded as a background and is therefore subtracted by the lock-in amplifier. Thus, this emission should not be included in the line-of-sight simulation.

It is clear from Fig. 20 that the emission of $\mathrm{CO}_{2}$ inside the spectrometer (blue curve) is responsible for the discrepancy between the measured and predicted spectra between 4.2 and $4.3 \mu \mathrm{m}$. The line-of-sight model was corrected by first solving the radiative transfer equation over a 6 -meter path for ambient $\mathrm{CO}_{2}$, and then subtracting the emission of ambient $\mathrm{CO}_{2}$ over a 3-meter path inside the spectrometer. This means that only the absorption inside the $3-\mathrm{m}$ of the spectrometer is taken into account. The correction (Fig. 20, red solid line) finally shows good agreement with the experimental spectrum in the $4.2-4.3 \mu \mathrm{m}$ region.

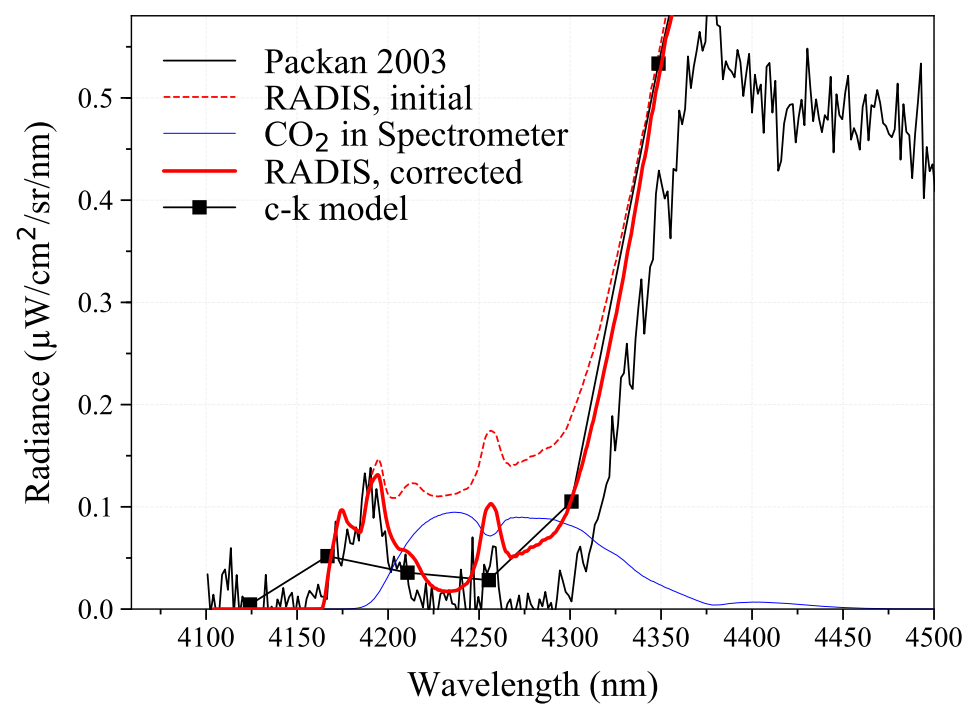

Figure 20: Experimental data (black, [25]), total emission along the line-of-sight (11 slabs $+6 \mathrm{~m}$ air) including the emission within the spectrometer (dashed red), contribution of $300 \mathrm{~K}$ emission within the spectrometer (blue), line-of-sight emission corrected from the spectrometer internal emission (solid, red), and predictions from the c-k model (black squares)

The discrepancy in the $4.4-4.7 \mu \mathrm{m}$ range can be explained from the contribution of the outer plasma slabs. We showed in Fig. 18 that the outer slabs, whose temperature is typically between 2000 and $3000 \mathrm{~K}$, have the highest volumetric power density. These slabs also correspond to the slabs where the signal from the $\mathrm{NO}(\mathrm{X}) 1 \rightarrow 0$ band used to infer the gas temperature is the weakest, hence their temperature is subject to the highest uncertainties.

Figure 22 shows the contribution of each individual slab to the total calculated radiance, for the adjusted temperature profile proposed in Figure 21, which features a smoother temperature drop to ambient temperature. The experimental and total radiance are also reproduced 
with an intensity scaled down by a factor of 10 . Figure 22 shows the contribution of all slabs, although only those circled in Fig. 21 are labeled (plasma slabs at 940, 1600, $2830 \mathrm{~K}$ and $3409 \mathrm{~K}$, and ambient $\mathrm{CO}_{2}$ outside of the spectrometer). The contribution is calculated as the emission of each slab transmitted through the remaining slabs along the line-of-sight. Fig. 22 first confirms that the emission of slabs having temperatures in the range $2000-$ $3000 \mathrm{~K}$ have a dominant contribution to the total emission of the torch. It also shows that the $4.4 \mu \mathrm{m}$ peak in the experimental spectrum is a characteristic feature of the contribution of a low temperature $\mathrm{CO}_{2}$ slab (for instance $940 \mathrm{~K}$ or $1600 \mathrm{~K}$ ). The presence of this feature in the experimental spectrum thus suggests that the temperature profile of Packan et al. [25] (Fig. 18) might be slightly overestimated at the outer radii where the error bars are large. The line-of-sight spectrum (Fig. 22, red) calculated with the adjusted profile of Fig. 21 yields much better agreement with the experimental spectrum.

We have shown that the RADIS line-of-sight model is able to reproduce the low signal experimental spectrum measured at the exit of the RF plasma torch using the measured temperature profile (Fig. 19). In order to achieve an even better agreement (Fig. 22), it was necessary to correct for the emission of ambient $\mathrm{CO}_{2}$ within the spectrometer, and to slightly adjust the temperature profile at the edges of the plasma. Indeed, these outer regions have the largest contribution to the emission but also the largest temperature uncertainties.

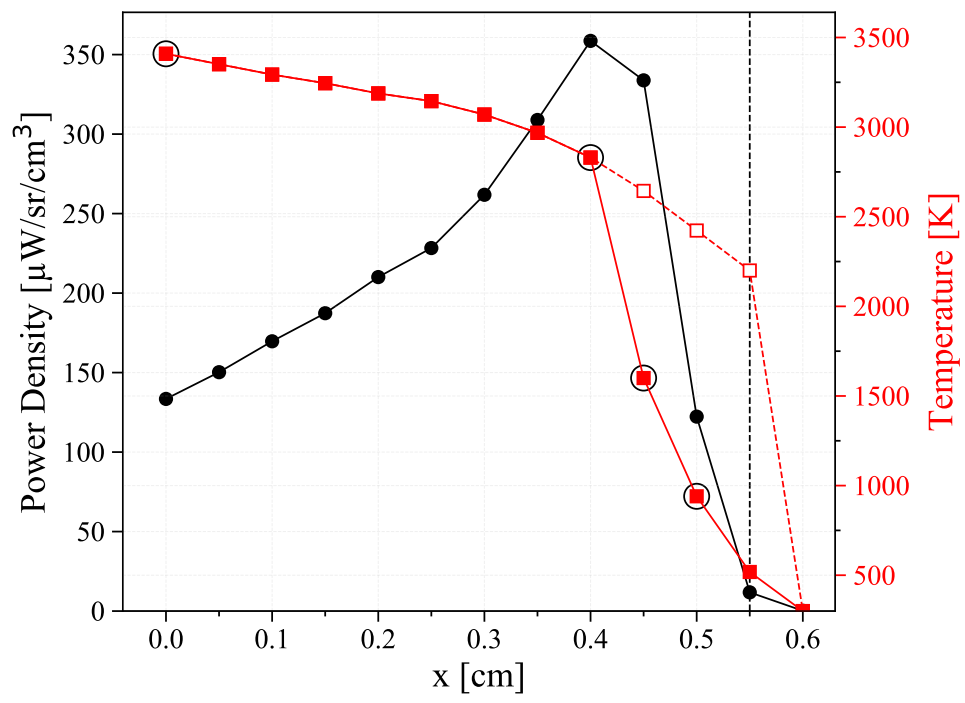

Figure 21: Adjusted temperature profile at the exit of the RF torch plasma torch (solid, red, filled), and power density of the $\mathrm{CO}_{2} 4.2 \mu \mathrm{m}$ band assuming chemical equilibrium in the plasma initially composed of air with $363 \mathrm{ppm}$ of $\mathrm{CO}_{2}$ (black). The circled points correspond to the black curves plotted in Fig. 22 The initial temperature profile of Fig. 18 is shown for reference (dashed, red, hollow). 


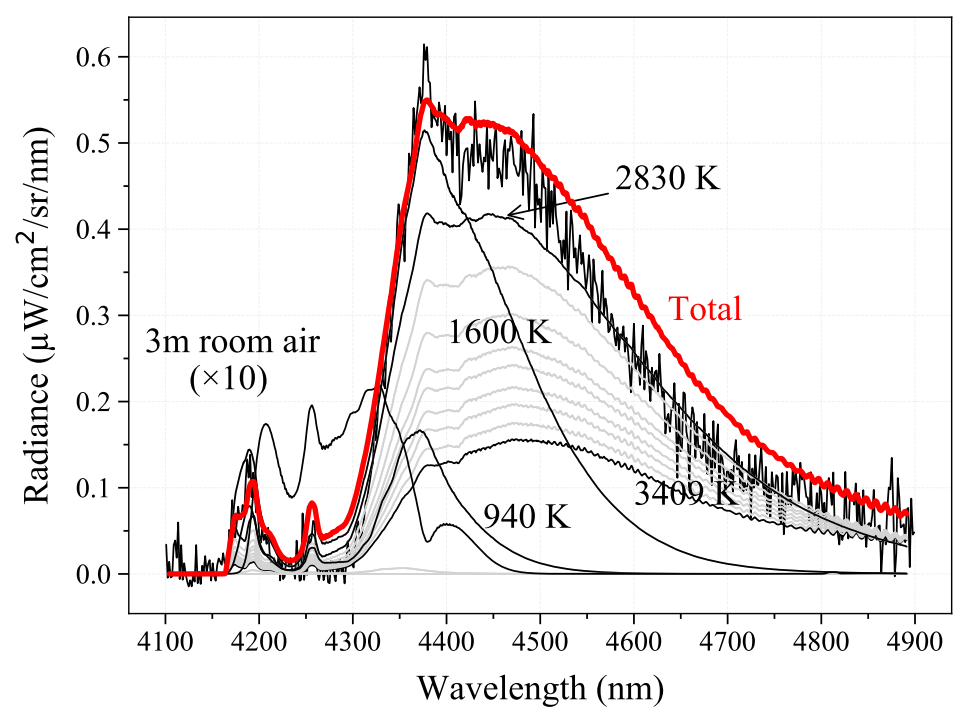

Figure 22: Total emission spectrum (thick, red) calculated with the temperature profile of Fig. 21 compared to the experimental spectrum (thick, black). The contribution of individual slabs to the total emission is shown with the intensity multiplied by 10, without a label (thin, gray) or with a label (thin, black) for the temperatures circled in Fig. 21.

\section{Conclusion}

RADIS, a radiative solver for infrared nonequilibrium spectra of polyatomic molecules, was presented. It uses line positions and intensities from HITRAN-like databases, and rovibrational energies from tabulated databases or calculated with Dunham expansions. The code can model any type of vibrational population distributions, including Boltzmann, Treanor or arbitrary distributions, with one or multiple vibrational temperatures. Non-homogeneous media can be modeled within the line-of-sight module.

Various optimization strategies managed to reduce computation times of large polyatomic spectra down to a few seconds with negligible errors, for instance $\mathrm{H}_{2} \mathrm{O}$ (Fig. 10), and $\mathrm{CH}_{4}$ (Fig. 9). The sensitivity to the different computation parameters were quantified separately: optimized values of the line broadening wing cutoff (3.4), and the line intensity cutoff (3.2) were given. The effect of the line database was also investigated (3.3) in the case of the $4.2 \mu \mathrm{m}$ band of $\mathrm{CO}_{2}$ : calculations with the HITEMP-2010 [26] database yielded good agreement with the CDSD-4000 [11] database up to temperatures of $2200 \mathrm{~K}$, yet were typically one to two orders of magnitude faster. The use of a pseudo-continuum (3.5) drastically reduced the number of lines resolved without any impact on the calculated spectra.

RADIS was compared to existing line-by-line codes (HAPI [5], Specair [3]) and experimental validation cases including infrared measurements from an equilibrium microwave torch [47], a low-pressure nonequilibrium laser discharge [34], and a NASA shock-tube experiment [48]. 
Good agreement with existing codes and experiments were obtained in all cases. Finally, RADIS was applied to improve the current modeling of an equilibrium RF plasma torch [25]. RADIS is distributed at [1] under a GNU Lesser General Public License (LGPLv3), i.e., it is available for use and modifications as long as modifications are described and licensed for free under LGPLv3.

\section{Acknowlegments}

The authors are very thankful to Dr. S.A.A Tashkun for providing the rovibrational energies of the ${ }^{12} \mathrm{C}^{16} \mathrm{O}^{16} \mathrm{O}$ isotope used in the comparison with the vibrating rotor model.

\section{References}

[1] E. Pannier, RADIS: a Nonequilibrium Infrared Radiative Solver: Source Code and User Guide, URL https://github.com/radis/radis, 2018.

[2] C. O. Laux, T. G. Spence, C. H. Kruger, R. N. Zare, Optical diagnostics of atmospheric pressure air plasmas, Plasma Sources Science and Technology 12 (2) (2003) 125, ISSN 0963-0252, doi:10.1088/0963$0252 / 12 / 2 / 301$.

[3] C. O. Laux, J. Andreasson, Specair 3.0, URL http://www.spectralfit.com, 2013.

[4] C. S. Goldenstein, V. A. Miller, R. Mitchell Spearrin, C. L. Strand, SpectraPlot.com: Integrated spectroscopic modeling of atomic and molecular gases, Journal of Quantitative Spectroscopy and Radiative Transfer 200 (2017) 249-257, ISSN 00224073, doi:10.1016/j.jqsrt.2017.06.007.

[5] R. Kochanov, I. Gordon, L. Rothman, P. Wcisło, C. Hill, J. Wilzewski, HITRAN Application Programming Interface (HAPI): A comprehensive approach to working with spectroscopic data, Journal of Quantitative Spectroscopy and Radiative Transfer 177 (2016) 15-30, ISSN 00224073, doi: 10.1016/j.jqsrt.2016.03.005.

[6] J. Voráč, P. Synek, L. Potočáková, J. Hnilica, V. Kudrle, Batch processing of overlapping molecular spectra as a tool for spatio-temporal diagnostics of power modulated microwave plasma jet, Plasma Sources Science and Technology 26 (2) (2017) 025010, ISSN 1361-6595, doi:10.1088/1361-6595/aa51f0.

[7] B. L. M. Klarenaar, R. Engeln, D. C. M. van den Bekerom, M. C. M. van de Sanden, A. S. MorilloCandas, O. Guaitella, Time evolution of vibrational temperatures in a CO 2 glow discharge measured with infrared absorption spectroscopy, Plasma Sources Science and Technology 26 (11) (2017) 115008, ISSN 1361-6595, doi:10.1088/1361-6595/aa902e.

[8] E. E. Whiting, P. Chul, Y. Liu, O. Arnold, A. Paterson, C. Park, Y. Liu, J. O. Arnold, J. a. Paterson, NEQAIR96, Nonequilibrium and Equilibrium Radiative Transport and Spectra Program: User's Manual, Tech. Rep., 1996.

[9] B. A. Cruden, A. M. Brandis, Updates to the NEQAIR Radiation Solver, in: International Workshop on Radiation of High Temperature Gases in Atmospheric Entry; 6th, 2014.

[10] G. Palmer, B. Cruden, Experimental Validation of CO2 Radiation Simulations, in: 43rd AIAA Thermophysics Conference, 2012-3188, American Institute of Aeronautics and Astronautics, Reston, Virigina, ISBN 978-1-62410-186-1, 1-15, doi:10.2514/6.2012-3188, 2012.

[11] S. A. Tashkun, V. I. Perevalov, CDSD-4000: High-resolution, high-temperature carbon dioxide spectroscopic databank, Journal of Quantitative Spectroscopy and Radiative Transfer 112 (9) (2011) 14031410, ISSN 00224073, doi:10.1016/j.jqsrt.2011.03.005. 
[12] S. Tashkun, V. Perevalov, J. Teffo, L. Rothman, V. Tyuterev, Global Fitting of 12C16O2 VibrationalRotational Line Positions Using the Effective Hamiltonian Approach, Journal of Quantitative Spectroscopy and Radiative Transfer 60 (5) (1998) 785-801, ISSN 00224073, doi:10.1016/S0022-4073(98)00082-X.

[13] C. O. Johnston, B. R. Hollis, K. Sutton, Spectrum Modeling for Air Shock-Layer Radiation at LunarReturn Conditions, Journal of Spacecraft and Rockets 45 (5) (2008) 865-878, ISSN 0022-4650, doi: 10.2514/1.33004.

[14] C. O. Johnston, A. M. Brandis, Modeling of nonequilibrium CO Fourth-Positive and CN Violet emission in CO2-N2gases, Journal of Quantitative Spectroscopy and Radiative Transfer 149 (2014) 303-317, ISSN 00224073, doi:10.1016/j.jqsrt.2014.08.025.

[15] P. Rivière, A. Soufiani, Updated band model parameters for $\mathrm{H} 2 \mathrm{O}, \mathrm{CO} 2, \mathrm{CH} 4$ and $\mathrm{CO}$ radiation at high temperature, International Journal of Heat and Mass Transfer 55 (13-14) (2012) 3349-3358, ISSN 00179310, doi:10.1016/j.ijheatmasstransfer.2012.03.019.

[16] A. Lemal, H. Takayanagi, S. Nomura, K. Fujita, Simulations of Carbon Dioxide Equilibrium Infrared Radiation Measurements, Journal of Thermophysics and Heat Transfer .

[17] H. Takayanagi, A. Lemal, S. Nomura, K. Fujita, Measurements of Carbon Dioxide Nonequilibrium Infrared Radiation in Shocked and Expanded Flows, Journal of Thermophysics and Heat Transfer 32 (2) (2018) 483-494, ISSN 0887-8722, doi:10.2514/1.T5200.

[18] C. Rond, A. Bultel, P. Boubert, B. G. Chéron, Spectroscopic measurements of nonequilibrium CO2 plasma in RF torch, Chemical Physics 354 (1-3) (2008) 16-26, ISSN 03010104, doi:10.1016/j.chemphys. 2008.09.006.

[19] H. Liebhart, M. Fertig, G. Herdrich, S. Fasoulas, Demonstration of the capabilities of PARADE with regard to the calculation of the linear tri-atomic molecule CO2 2010 (February) (2011) 12-15.

[20] M. Lino da Silva, B. Lopez, S. Espinho, SPARTAN 2.6 Users manual .

[21] M. Lino da Silva, D. Vacher, M. Dudeck, P. André, G. Faure, Radiation from an equilibrium CO2 N2 plasma in the [250850 nm] spectral region: II. Spectral modelling, Plasma Sources Science and Technology 17 (3) (2008) 035013, ISSN 0963-0252, doi:10.1088/0963-0252/17/3/035013.

[22] A. A. Kutepov, L. Rezac, A. G. Feofilov, Evidence of a significant rotational non-LTE effect in the CO2 4.3 ţm PFS-MEX limb spectra, Atmospheric Measurement Techniques 10 (1) (2017) 265-271, ISSN 1867-8548, doi:10.5194/amt-10-265-2017.

[23] M. López-Puertas, M. A. López-Valverde, C. P. Rinsland, M. R. Gunson, Analysis of the upper atmosphere CO2 (v2) vibrational temperatures retrieved from ATMOS/Spacelab 3 observations, Journal of Geophysical Research 97 (D18) (1992) 20469, ISSN 0148-0227, doi:10.1029/92JD02026.

[24] E. Pannier, V. Baillard, C. O. Laux, In-situ measurements of the vibrational excitation of $\mathrm{CO} 2$ by nanosecond discharges at atmospheric pressure (In Preparation) .

[25] D. Packan, C. O. Laux, R. J. Gessman, L. Pierrot, C. H. Kruger, Measurement and Modeling of OH, NO, and CO Infrared Radiation at $3400 \mathrm{~K}$, Journal of Thermophysics and Heat Transfer 17 (4) (2003) 450-456, ISSN 0887-8722, doi:10.2514/2.6803.

[26] L. S. Rothman, I. E. Gordon, R. J. Barber, H. Dothe, R. R. Gamache, A. Goldman, V. I. Perevalov, S. A. Tashkun, J. Tennyson, HITEMP, the high-temperature molecular spectroscopic database, Journal of Quantitative Spectroscopy and Radiative Transfer 111 (15) (2010) 2139-2150, ISSN 00224073, doi: 10.1016/j.jqsrt.2010.05.001.

[27] L. Rothman, C. Rinsland, A. Goldman, S. Massie, D. Edwards, J.-M. Flaud, A. Perrin, C. Camy-Peyret, V. Dana, J.-Y. Mandin, J. Schroeder, A. McCann, R. Gamache, R. Wattson, K. Yoshino, K. Chance, K. Jucks, L. Brown, V. Nemtchinov, P. Varanasi, the Hitran Molecular Spectroscopic Database and Hawks (Hitran Atmospheric Workstation): 1996 Edition, Journal of Quantitative Spectroscopy and Radiative Transfer 60 (5) (1998) 665-710, ISSN 00224073, doi:10.1016/S0022-4073(98)00078-8.

[28] J. Fischer, R. Gamache, a. Goldman, L. Rothman, A. Perrin, Total internal partition sums for molecular species in the 2000 edition of the HITRAN database, Journal of Quantitative Spectroscopy and Radiative Transfer 82 (1-4) (2003) 401-412, ISSN 00224073, doi:10.1016/S0022-4073(03)00166-3.

[29] S. A. Tashkun, C12O16O16 energy levels (private communication), 2017.

[30] P. E. Ciddor, Refractive index of air: new equations for the visible and near infrared., Applied optics 
35 (9) (1996) 1566-1573, ISSN 0003-6935, doi:10.1364/AO.35.001566.

[31] E. Whiting, An empirical approximation to the Voigt profile, Journal of Quantitative Spectroscopy and Radiative Transfer 8 (6) (1968) 1379-1384, ISSN 00224073, doi:10.1016/0022-4073(68)90081-2.

[32] J. Olivero, R. Longbothum, Empirical fits to the Voigt line width: A brief review, Journal of Quantitative Spectroscopy and Radiative Transfer 17 (2) (1977) 233-236, ISSN 00224073, doi: 10.1016/0022-4073(77)90161-3.

[33] M. Šimečková, D. Jacquemart, L. S. Rothman, R. R. Gamache, A. Goldman, Einstein A-coefficients and statistical weights for molecular absorption transitions in the HITRAN database, Journal of Quantitative Spectroscopy and Radiative Transfer 98 (1) (2006) 130-155, ISSN 00224073, doi: 10.1016/j.jqsrt.2005.07.003.

[34] C. Dang, J. Reid, B. K. Garside, Detailed vibrational population distributions in a CO2 laser discharge as measured with a tunable diode laser, Applied Physics B Photophysics and Laser Chemistry 27 (3) (1982) 145-151, ISSN 07217269, doi:10.1007/BF00694640.

[35] R. Koopmann, A. Saunders, Nonequilibrium population distributions for vibrational energy levels of $\mathrm{CO} 2$ in CO-O2 flames at reduced pressure, Journal of Quantitative Spectroscopy and Radiative Transfer 10 (5) (1970) 403-421, ISSN 00224073, doi:10.1016/0022-4073(70)90106-8.

[36] W. J. Witteman, The CO laser, Springer-Verlag, ISBN 3540477446, 1987.

[37] G. Guelachvili, D. de Villeneuve, R. Farrenq, W. Urban, J. Verges, Dunham coefficients for seven isotopic species of CO, Journal of Molecular Spectroscopy 98 (1) (1983) 64-79, ISSN 1096083X, doi: 10.1016/0022-2852(83)90203-5.

[38] A. W. Mantz, J. P. Maillard, W. B. Roh, K. Narahari Rao, Ground state molecular constants of 12C16O, Journal of Molecular Spectroscopy 57 (1) (1975) 155-159, ISSN 1096083X, doi:10.1016/00222852(75)90049-1.

[39] A. P. Mishra, B. J. Shetty, R. J. Kshirsagar, Fourier transform emission spectroscopy of $\Delta \mathrm{v}=2$ sequence bands of the $\mathrm{CO}$ molecule in the ground electronic state, Journal of Molecular Spectroscopy 232 (2) (2005) 296-307, ISSN 00222852, doi:10.1016/j.jms.2005.05.002.

[40] Y. Babou, P. Rivière, M.-Y. Perrin, A. Soufiani, High-Temperature and Nonequilibrium Partition Function and Thermodynamic Data of Diatomic Molecules, International Journal of Thermophysics 30 (2) (2009) 416-438, ISSN 0195-928X, doi:10.1007/s10765-007-0288-6.

[41] E. Pannier, C. O. Laux, Limits of CO2 Nonequilibrium Spectral Models (In Preparation) .

[42] A. Spielfiedel, N. Feautrier, C. Cossart-Magos, G. Chambaud, P. Rosmus, H.-J. H. Werner, P. Botschwina, C. CossartMagos, G. Chambaud, P. Rosmus, H.-J. H. Werner, P. Botschwina, Bent valence excited states of CO2, The Journal of Chemical Physics 97 (11) (1992) 8382, ISSN 00219606, doi:10.1063/1.463408.

[43] M. L. da Silva, SPARTAN: Simulation of PlasmA Radiation in ThermodynAmic Nonequilibrium, URL http://esther.ist.utl.pt/spartan/, 2016.

[44] I. Gordon, L. Rothman, C. Hill, R. Kochanov, Y. Tan, P. Bernath, V. Boudon, A. Campargue, B. Drouin, J. M. Flaud, R. Gamache, J. Hodges, V. Perevalov, K. Shine, M.-a. Smith, The HITRAN2016 Molecular Spectroscopic Database, Journal of Quantitative Spectroscopy and Radiative Transfer 6 (38) (2017) 3-69, ISSN 00224073, doi:10.1016/j.jqsrt.2017.06.038.

[45] J. O. Arnold, D. M. Cooper, C. Park, G. K. S. Prakash, Line-by-Line Transport Calculations for Jupiter Entry Probes, in: Entry Heating and Thermal Protection, American Institute of Aeronautics and Astronautics, New York, 52-82, doi:10.2514/5.9781600865435.0052.0082, 1980.

[46] S. Depraz, M. Perrin, A. Soufiani, Infrared emission spectroscopy of CO2 at high temperature. Part I: Experimental setup and source characterization, Journal of Quantitative Spectroscopy and Radiative Transfer 113 (1) (2012) 1-13, ISSN 00224073, doi:10.1016/j.jqsrt.2011.09.002.

[47] S. Depraz, M. Perrin, P. Rivière, A. Soufiani, Infrared emission spectroscopy of CO2 at high temperature. Part II: Experimental results and comparisons with spectroscopic databases, Journal of Quantitative Spectroscopy and Radiative Transfer 113 (1) (2012) 14-25, ISSN 00224073, doi: 10.1016/j.jqsrt.2011.09.013.

[48] B. A. Cruden, A. M. Brandis, D. K. Prabhu, Measurement and Characterization of Mid-wave Infrared 
Radiation in CO2 Shocks, in: 11th AIAA/ASME Joint Thermophysics and Heat Transfer Conference, American Institute of Aeronautics and Astronautics, Reston, Virginia, ISBN 978-1-62410-281-3, doi: 10.2514/6.2014-2962, 2014.

[49] NASA, CEARUN, URL https://cearun.grc.nasa.gov/, 1971.

[50] L. Pierrot, P. Rivière, A. Soufiani, J. Taine, A fictitious-gas-based absorption distribution function global model for radiative transfer in hot gases, Journal of Quantitative Spectroscopy and Radiative Transfer 62 (1999) 609-624, ISSN 00224073, doi:10.1016/S0022-4073(98)00124-1. 